\title{
What Is a Simple Liquid?
}

\author{
Trond S. Ingebrigtsen, Thomas B. Schrøder, and Jeppe C. Dyre* \\ DNRF Centre "Glass and Time," IMFUFA, Department of Sciences, Roskilde University, Postbox 260, DK-4000 Roskilde, Denmark
} (Received 16 November 2011; published 15 March 2012)

This paper is an attempt to identify the real essence of simplicity of liquids in John Locke's understanding of the term. Simple liquids are traditionally defined as many-body systems of classical particles interacting via radially symmetric pair potentials. We suggest that a simple liquid should be defined instead by the property of having strong correlations between virial and potential-energy equilibrium fluctuations in the $N V T$ ensemble. There is considerable overlap between the two definitions, but also some notable differences. For instance, in the new definition simplicity is not a direct property of the intermolecular potential because a liquid is usually only strongly correlating in part of its phase diagram. Moreover, not all simple liquids are atomic (i.e., with radially symmetric pair potentials) and not all atomic liquids are simple. The main part of the paper motivates the new definition of liquid simplicity by presenting evidence that a liquid is strongly correlating if and only if its intermolecular interactions may be ignored beyond the first coordination shell (FCS). This is demonstrated by $N V T$ simulations of the structure and dynamics of several atomic and three molecular model liquids with a shifted-forces cutoff placed at the first minimum of the radial distribution function. The liquids studied are inverse power-law systems ( $r^{-n}$ pair potentials with $\left.n=18,6,4\right)$, Lennard-Jones (LJ) models (the standard LJ model, two generalized Kob-Andersen binary LJ mixtures, and the Wahnstrom binary LJ mixture), the Buckingham model, the Dzugutov model, the LJ Gaussian model, the Gaussian core model, the Hansen-McDonald molten salt model, the Lewis-Wahnstrom ortho-terphenyl model, the asymmetric dumbbell model, and the single-point charge water model. The final part of the paper summarizes properties of strongly correlating liquids, emphasizing that these are simpler than liquids in general. Simple liquids, as defined here, may be characterized in three quite different ways: (1) chemically by the fact that the liquid's properties are fully determined by interactions from the molecules within the FCS, (2) physically by the fact that there are isomorphs in the phase diagram, i.e., curves along which several properties like excess entropy, structure, and dynamics, are invariant in reduced units, and (3) mathematically by the fact that throughout the phase diagram the reduced-coordinate constant-potential-energy hypersurfaces define a one-parameter family of compact Riemannian manifolds. No proof is given that the chemical characterization follows from the strong correlation property, but we show that this FCS characterization is consistent with the existence of isomorphs in strongly correlating liquids' phase diagram. Finally, we note that the FCS characterization of simple liquids calls into question the physical basis of standard perturbation theory, according to which the repulsive and attractive forces play fundamentally different roles for the physics of liquids.

DOI: 10.1103/PhysRevX.2.011011 Subject Areas: Chemical Physics, Condensed Matter Physics, Statistical Physics

\section{INTRODUCTION}

Going back to Plato, classification or categorization is the epistemological process that groups objects based on similar properties [1]. Having primarily biological examples in mind, Aristotle defined categories as discrete entities characterized by properties shared by their members [2]. Aristotle, and Locke in 1690 in much more detail, distinguished between the nominal essence and the real essence of an object [3]. The nominal essence comes from experience and represents the object's appearance, whereas the real essence represents the object's deeper, constituting

\section{*dyre@ruc.dk}

Published by the American Physical Society under the terms of the Creative Commons Attribution 3.0 License. Further distribution of this work must maintain attribution to the author(s) and the published article's title, journal citation, and DOI. features. For instance, the real essence of a material thing is its atomic constitution, because this is the causal basis of all the thing's observable properties [4]. A scientific classification is particularly useful if it reflects the real essence of the objects in question by identifying their underlying common features, from which the more obvious and easily observable nominal properties follow. Having in mind Locke's concept of real essence, we argue below for a new definition of the class of simple liquids.

Physicists love simple systems. This reflects the fundamental paradigm that, in order to capture a given phenomenon, simpler is better. Most classifications in physics are clear-cut, for example, the classification of elementary particles into baryons and leptons, whereas classifications in other sciences usually have a wealth of borderline cases. Because of the diversity of molecules, it is reasonable to expect a definition of "simple liquids" to be of the latter type. 
The concept of a simple liquid is old, but it remains central as evidenced by the 2003 book entitled Basic Concepts for Simple and Complex Liquids [5] or the review of nonsimple liquids entitled Theory of Complicated Liquids from 2007 [6]. Generations of liquid-state theorists were introduced to this exciting topic by studying Hansen and McDonald's textbook Theory of Simple Liquids [7]. This book first appeared in 1976, following a period of spectacular progress in the theory of liquids, catalyzed by some of the very first scientific computer simulations.

In Ref. [7] a simple liquid is defined as a classical system of approximately spherical, nonpolar molecules interacting via pair potentials. This and closely related definitions of liquid simplicity have been standard for many years [8-12]. In this definition, simple liquids have much in common with the chemists' "nonassociated liquids" [13], but there are some significant differences. Chemists generally regard a liquid as simple even if it consists of elongated molecules, as long as these are without internal degrees of freedom and interact primarily via van der Waals forces. Many physicists would probably disagree. Thus, it is far from trivial to ask: What characterizes a simple liquid? More accurately: Given a classical system of rigid bodies with potential energy as a function of the bodies' centers of masses and their spatial orientations, is it possible to give a quantitative criterion for how simple the system is? If yes, is simplicity encoded uniquely in the potential-energy function or may the degree of simplicity vary throughout the phase diagram?

Recent works identified and described the properties of what we have termed strongly correlating liquids [14-25]. By definition, in these liquids the virial $W$ and the potential energy $U$ correlate strongly in their constant-volume thermal-equilibrium fluctuations. Recall that the average virial $\langle W\rangle$ gives the contribution to the pressure from intermolecular interactions, which is added to the ideal-gas term $N k_{B} T$, deriving from momentum transport via particle motion (below $p$ is the pressure, $V$ the volume, $N$ the number of particles, $k_{B}$ Boltzmann's constant, and $T$ the temperature):

$$
p V=N k_{B} T+\langle W\rangle \text {. }
$$

The term strongly correlating liquid refers to the case when the $W U$ correlation coefficient in the $N V T$ ensemble is larger than 0.9 [17]. If angular brackets denote an $N V T$ ensemble average, the correlation coefficient $R$ is defined by

$$
R=\frac{\langle\Delta W \Delta U\rangle}{\sqrt{\left\langle(\Delta W)^{2}\right\rangle\left\langle(\Delta U)^{2}\right\rangle}} .
$$

An example of a strongly correlating liquid is the standard Lennard-Jones (LJ) liquid at typical condensed-phase state points. Many other systems, including some molecular models, have been shown to be strongly correlating; we refer the reader to papers that derive and document the several simple properties of strongly correlating liquids [14-25]. These properties are summarized briefly in Sec. IVA after the presentation of the simulation results.

The present work is motivated by developments initiated by recent findings by Berthier and Tarjus [26,27]. These authors showed that for the viscous Kob-Andersen binary Lennard-Jones mixture $[28,29]$ the dynamics is not reproduced properly by cutting the potentials at their minima according to the well-known Weeks-Chandler-Andersen (WCA) recipe [30]. The role of the cutoff was subsequently studied in two papers [31,32], showing that placing a shifted-forces cutoff at the first minimum of the pair correlation function-thus defining the first coordination shell (FCS)_-gives good results for Lennard-Jones-type systems. This is the case not only at moderate densities, but also at very high densities. Applying the same cutoff to water does not work properly [33]. Water is an example of a nonstrongly correlating liquid with $R \approx 0$ at ambient conditions, a consequence of water's density maximum [17]. These findings led us to speculate whether it is a general property of strongly correlating liquids that the intermolecular interactions may be ignored beyond the FCS without compromising accuracy to any significant extent. The main part of this paper shows that, indeed, using such an "FCS cutoff" gives accurate simulation results if the liquid is strongly correlating.

The paper presents results obtained from computer simulations of several different systems, only some of which are strongly correlating. We investigate the role of the FCS in determining liquid structure and dynamics. Structure is probed by the radial distribution function (RDF), dynamics by the incoherent or, in a few cases, coherent intermediate scattering function (ISF) at the wave vector defined by the maximum of the static structure factor. The numerical evidence is clear. By varying the cutoff of the intermolecular forces, we find that in order to get accurate simulation results it is enough to take into account merely the interactions within the FCS if and only if the liquid is strongly correlating. In other words, for strongly correlating liquids, interactions beyond the FCS are unimportant, and this applies only for these liquids. At present there are no compelling arguments for this empirical "FCS property," but we argue briefly in Sec. IV B that it is consistent with known properties of strongly correlating liquids.

The FCS property of strongly correlating liquids shows that these are simpler than liquids in general. A number of other simple properties of strongly correlating liquids have been identified previously [14-25]. Altogether, these facts motivate our new definition of liquid simplicity.

Section II presents the results from molecular dynamics simulations and Sec. III summarizes the results. Section IV gives an overview of the many simple properties of strongly correlating liquids, motivating our suggestion that a liquid is to be defined as simple if it is strongly correlating at the state point in question. Section $\mathrm{V}$ gives a few concluding remarks. 


\section{MOLECULAR DYNAMICS SIMULATIONS OF ATOMIC AND MOLECULAR LIQUIDS}

In a computer simulation, the interactions, which usually extend in principle to infinity, are truncated at some cutoff distance $r_{c}$ beyond which they are ignored. To avoid a discontinuity in the force, which can severely affect the results $[31,34]$, the simulations reported below use potentials modified such that the force goes continuously to zero at $r_{c}$. This is done by applying a so-called shifted-forces (SF) cutoff [34-36] where, if the pair potential is $v(r)$ and the pair force is $f(r)=-v^{\prime}(r)$, the shifted force is given by

$$
f_{\mathrm{SF}}(r)= \begin{cases}f(r)-f\left(r_{c}\right) & \text { if } r<r_{c} \\ 0 & \text { if } r>r_{c} .\end{cases}
$$

This corresponds to using the following pair potential below $r_{c}: v_{\mathrm{SF}}(r)=v(r)-v^{\prime}\left(r_{c}\right)\left(r-r_{c}\right)-v\left(r_{c}\right)$. Using a SF cutoff gives more accurate results and better numerical stability than using the standard shifted-potential (SP) cutoff [31]. This is so despite the fact that a SF cutoff does not have the correct pair force for any $r$, whereas the pair force is correct below $r_{c}$ for a SP cutoff. Apparently, avoiding discontinuity of the force at $r_{c}$ is more important than maintaining the correct force. It was recently discussed [18] why adding a linear term to the pair potential affects neither structure nor dynamics to any significant extent. The reason is that, when one nearest-neighbor distance decreases, others increase in such a way that their sum is virtually constant. This argument is exact in one dimension and holds to a good approximation in 3D constant-volume simulations [18] (in constant-pressure simulations the volume fluctuates and the argument no longer applies). Coulomb interactions have also been treated by the SF cutoff procedure. Although the Coulomb interaction is long ranged and conditionally convergent, when $r_{c}$ is sufficiently large, a SF cutoff gives results close to those of the standard, much more involved, Ewald summation method $[37,38]$.

All simulations were performed in the $N V T$ ensemble with periodic boundary conditions using the Nose-Hoover algorithm [39-41]. We used the Roskilde University Molecular Dynamics package developed for state-of-theart graphics processing unit (GPU) computing [42]. For the molecular models, bond lengths were held fixed using the time-symmetrical central-difference algorithm [43-45].

The effect on the structure and dynamics of varying the pair-potential cutoff $r_{c}$ was recently investigated for the single-component Lennard-Jones liquid and the KobAndersen binary LJ mixture [31,32]. For both systems, it was found that if a SF cutoff is applied instead of the commonly used SP cutoff, the standard cutoff $r_{c}=2.5 \sigma$ can be decreased to $1.5 \sigma$ and still give the correct physics. The value $r_{c}=1.5 \sigma$ is close to the first minimum of the $\mathrm{RDF}$, implying that all nearest-neighbor interactions are accounted for. Decreasing the cutoff further quickly affects the simulations, an effect that is quite pronounced for the dynamics in the viscous regime [26,27].

In the following we investigate, for several systems, whether it is possible to choose a FCS cutoff and still get the correct physics. We start by studying strongly correlating atomic liquids. Then, data are presented for a few atomic liquids that are not strongly correlating. Finally, data are given for two strongly correlating molecular liquids and a water model. Details of the models studied, the number of particles, etc., are given in Appendix A.

\section{A. Three inverse-power-law fluids}

We consider first systems with $100 \%$ correlation between virial and potential-energy equilibrium fluctuations in the $N V T$ ensemble. It follows from the definition of the virial $W=-1 / 3 \sum \mathbf{r}_{i} \cdot \nabla_{i} U$ [34] that a necessary and sufficient condition for $W$ to correlate perfectly with $U$ is that the potential energy is an Euler homogeneous function of the particle coordinates $\mathbf{r}_{i}$. This is clearly the case for systems with inverse power-law (IPL) pair potentials $\left[v(r) \propto r^{-n}\right]$, but note that potentials with nontrivial angular dependence may also be Euler homogeneous.

We simulated single-component IPL pair-potential systems with exponents $n=18,6,4$ at density $\rho=0.85$. Each system was studied at two temperatures. The simulated systems range from $n=18$, which is very harsh and repulsive, to $n=4$, which is quite soft and long ranged. The role of the cutoff is investigated by choosing three different, fairly small cutoffs: one placed at the first minimum of the RDF (red), one corresponding to the half height of the RDF from its minimum to its maximum (blue), and one placed to the right of the RDF first minimum (green), displaced the same amount as the difference between the first and the second cutoff.

The RDFs $g(r)$ are shown for $n=18,6,4$ in Fig. 1; $n=12$ gives similar results (not shown). The simulations with a SF cutoff at the first minimum of the RDF-referred to as FCS-cutoff simulations-give a faithful representation of the structure. The insets show, as functions of the cutoff, the deviations in RDF between the results for a FCS cutoff and the "true" large-cutoff results, quantified by integrating the numerical difference in the pair correlation function. Clearly, deviations increase sharply when the cutoff enters the FCS (blue crosses).

We simulated also the $n=3$ and $n=1$ IPL fluids. For both systems, a FCS cutoff does not lead to the correct physics. Both models do not have a proper thermodynamic limit, for which the exponent must be larger than the dimension [46]. For the $n=1 \mathrm{IPL}$ (Coulomb) fluid, this problem may be solved by introducing a uniform, neutralizing background of opposite charges, resulting in the well-known onecomponent plasma model [47]. An indication that a FCS cutoff works poorly when the IPL exponent approaches the dimension is seen for the $n=4$ simulation, for which the $W U$ correlation coefficient for the FCS cutoff starts to deviate significantly from unity. Moreover, but almost 

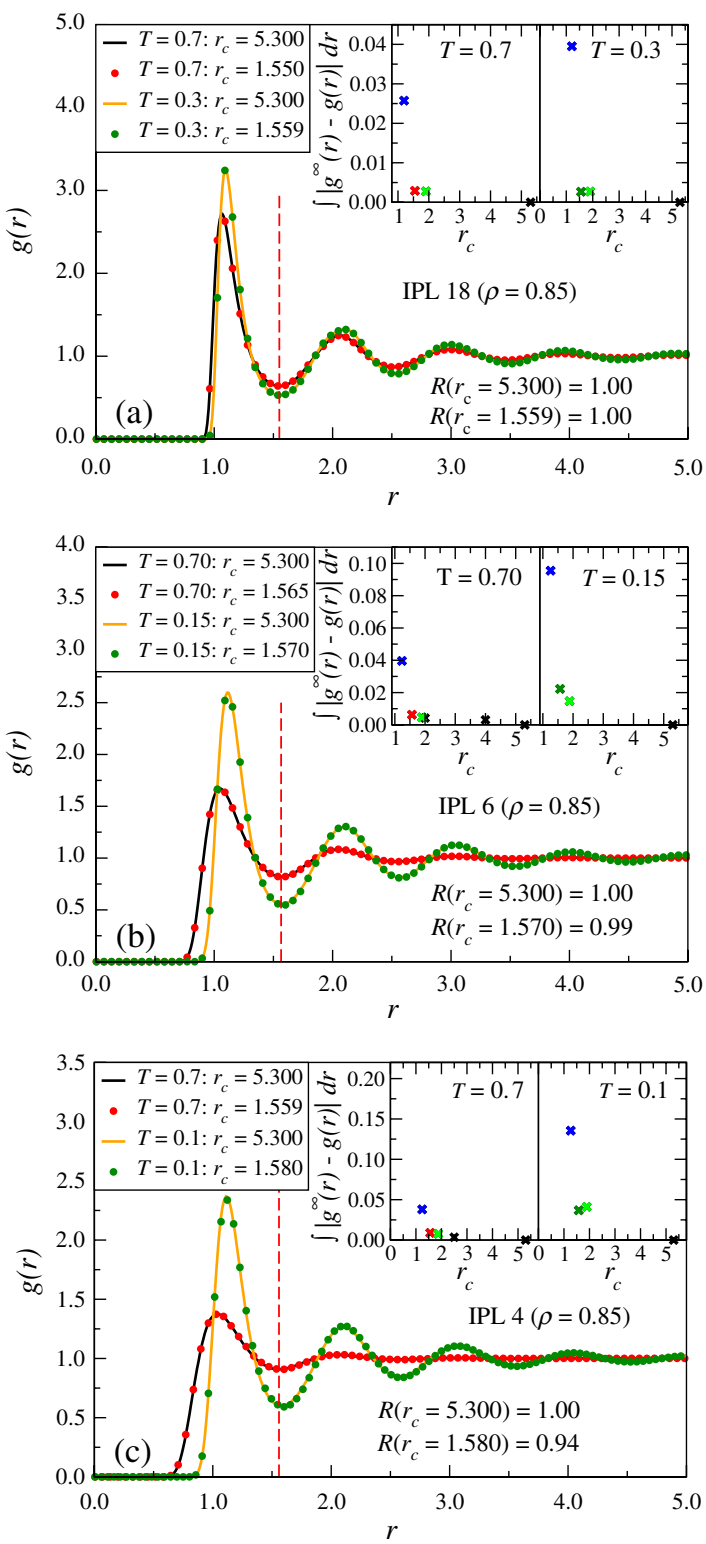

FIG. 1. RDFs for single-component IPL fluids with exponents $n=18,6,4$, each simulated at two temperatures at density $\rho=0.85$. The black and orange curves show reference simulation results with large cutoffs representing the true IPL behavior, the red and green dots give results from simulations with a FCS cutoff (marked by the vertical red dashed lines). The insets quantify the deviations in the RDF from the reference RDF as functions of the cutoff; deviations increase dramatically when the cutoff enters the FCS (blue crosses). In panels (a), (b), and (c) the virial potential-energy correlation coefficient $R$ is given for the lowest temperature (this quantity is exactly unity for IPL systems with infinite cutoff).

invisible in the figure, the $n=4$ pair correlation function's first maximum deviates slightly when comparing FCS and true simulations.

Figure 2 shows the incoherent ISFs evaluated at the wave vector corresponding to the first maximum of the static structure factor for the low-temperature state points
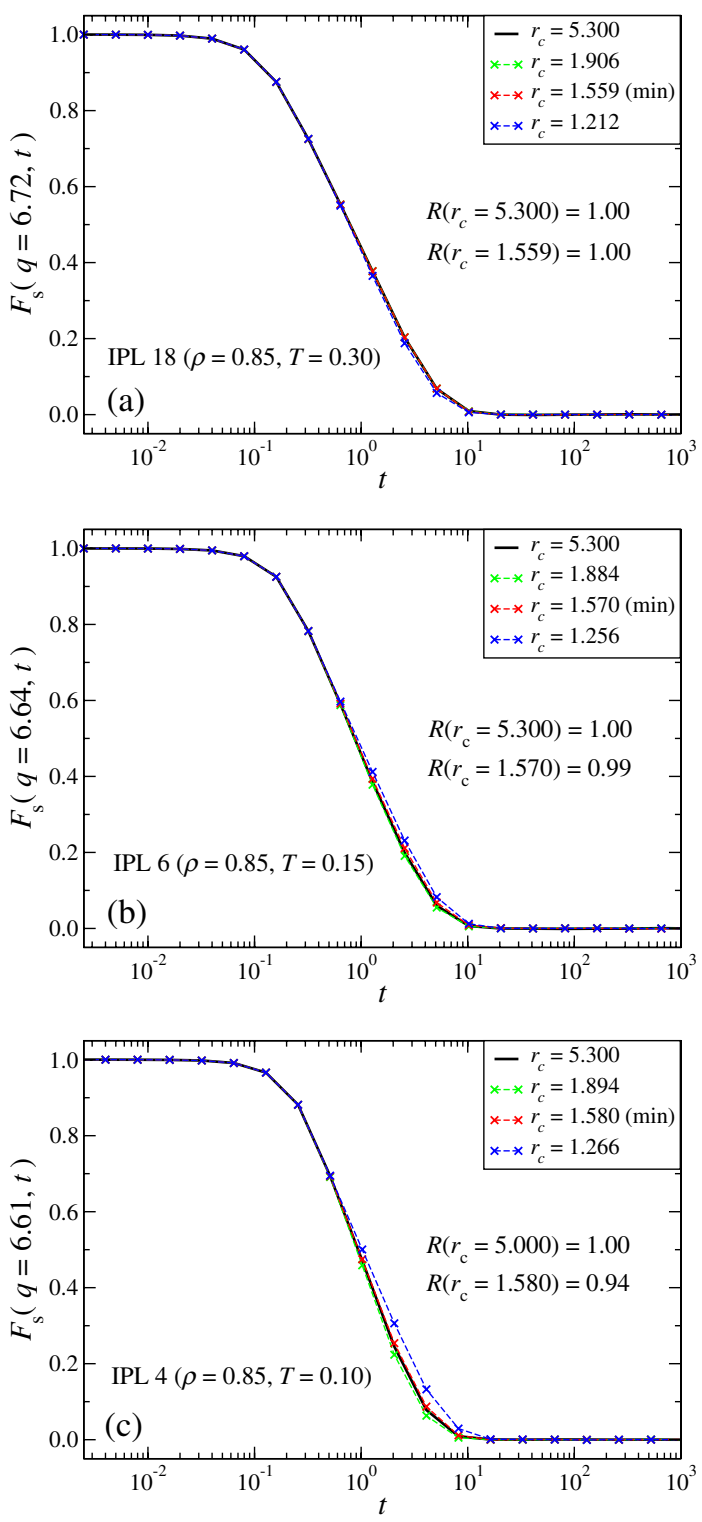

FIG. 2. Incoherent ISFs for the IPL fluids at the lowesttemperature state points of Fig. 1. The black curves give results for a large cutoff, the red crosses for a FCS cutoff (marked by the vertical red dashed lines in Fig. 1). (a) $n=18, T=0.30$; (b) $n=6, T=0.15$; (c) $n=4, T=0.10$.

of each of the three IPL systems. A good representation of the dynamics is obtained for all systems when the FCS cutoff is used.

\section{B. Lennard-Jones liquids}

Next, we consider what is probably the most studied potential in the history of computer simulations, the LJ pair potential,

$$
v_{\mathrm{LJ}}(r)=4 \epsilon\left[\left(\frac{\sigma}{r}\right)^{12}-\left(\frac{\sigma}{r}\right)^{6}\right] .
$$

Here, $\sigma$ and $\epsilon$ define, respectively, the length and energy scale of the interaction (dimensionless units defined by 


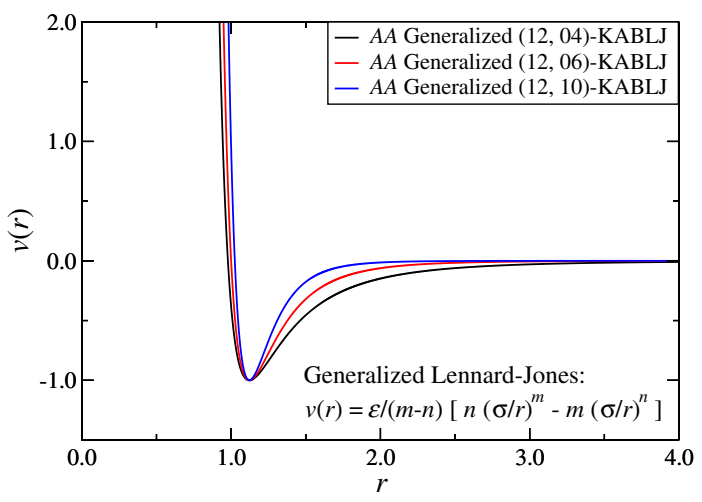

FIG. 3. The $A A$-particle generalized KABLJ pair potentials with fixed repulsive exponent 12 and three different attractive exponents $n=4,6,10$. The model parameters are given in Appendix A.

$\sigma=\epsilon=1$ are used below). This potential does not have $100 \%$ virial potential-energy correlations, but has still quite strong correlations with correlation coefficients $R>0.9$ in the condensed-fluid part of the phase diagram (and also in the crystalline phase [18]). We studied the single-component LJ (SCLJ) liquid, two generalized 80/20 Kob-Andersen binary LJ (KABLJ) mixtures with repulsive exponent 12 and attractive exponents $n=4,10$, and the Wahnstrom 50/50 binary LJ mixture (Fig. 3 and Appendix A give model details). The influence of a SF cutoff on simulation accuracy was investigated recently for the SCLJ liquid and the standard KABLJ mixture $(n=6)$ $[31,32]$, but for completeness we include results for the SCLJ system here as well. See also Table I.

The role of the cutoff is again investigated by choosing three different cutoffs: one placed at the first minimum of the RDF (red color in Figs. 4-8), one corresponding to the half height of the RDF from its minimum to its maximum (blue color in Figs. 4-8), and one displaced to the right of the minimum by the same amount as the difference between the first and the second cutoff (green color in Figs. 4-8).

In Fig. 4, RDFs are shown for the SCLJ liquid at three different state points. The red circles and curve show results from simulations with a FCS cutoff (marked by the vertical red dashed line); the black curves show the corresponding

TABLE I. The results for five state points of the SCLJ liquid. For each state point is given density, temperature, correlation coefficient, maximum deviation from the true RDF using a FCS cutoff, and maximum deviation from the true ISF using a FCS cutoff. The deviations clearly increase as the $W U$ correlation decreases.

\begin{tabular}{llllll}
\hline \hline System & $\rho$ & $T$ & $R$ & $|\Delta \mathrm{RDF}|_{\max }$ & $|\Delta \mathrm{ISF}|_{\max }$ \\
\hline SCLJ & 0.85 & 1.00 & 0.97 & $1.31 \times 10^{-2}$ & $5.10 \times 10^{-3}$ \\
SCLJ & 0.85 & 0.70 & 0.96 & $1.68 \times 10^{-2}$ & $8.28 \times 10^{-3}$ \\
SCLJ & 0.85 & 0.65 & 0.96 & $1.63 \times 10^{-2}$ & $8.96 \times 10^{-3}$ \\
SCLJ & 0.50 & 1.50 & 0.69 & $11.2 \times 10^{-2}$ & $7.94 \times 10^{-3}$ \\
SCLJ & 0.55 & 1.13 & 0.50 & $15.2 \times 10^{-2}$ & $12.0 \times 10^{-3}$ \\
\hline \hline
\end{tabular}
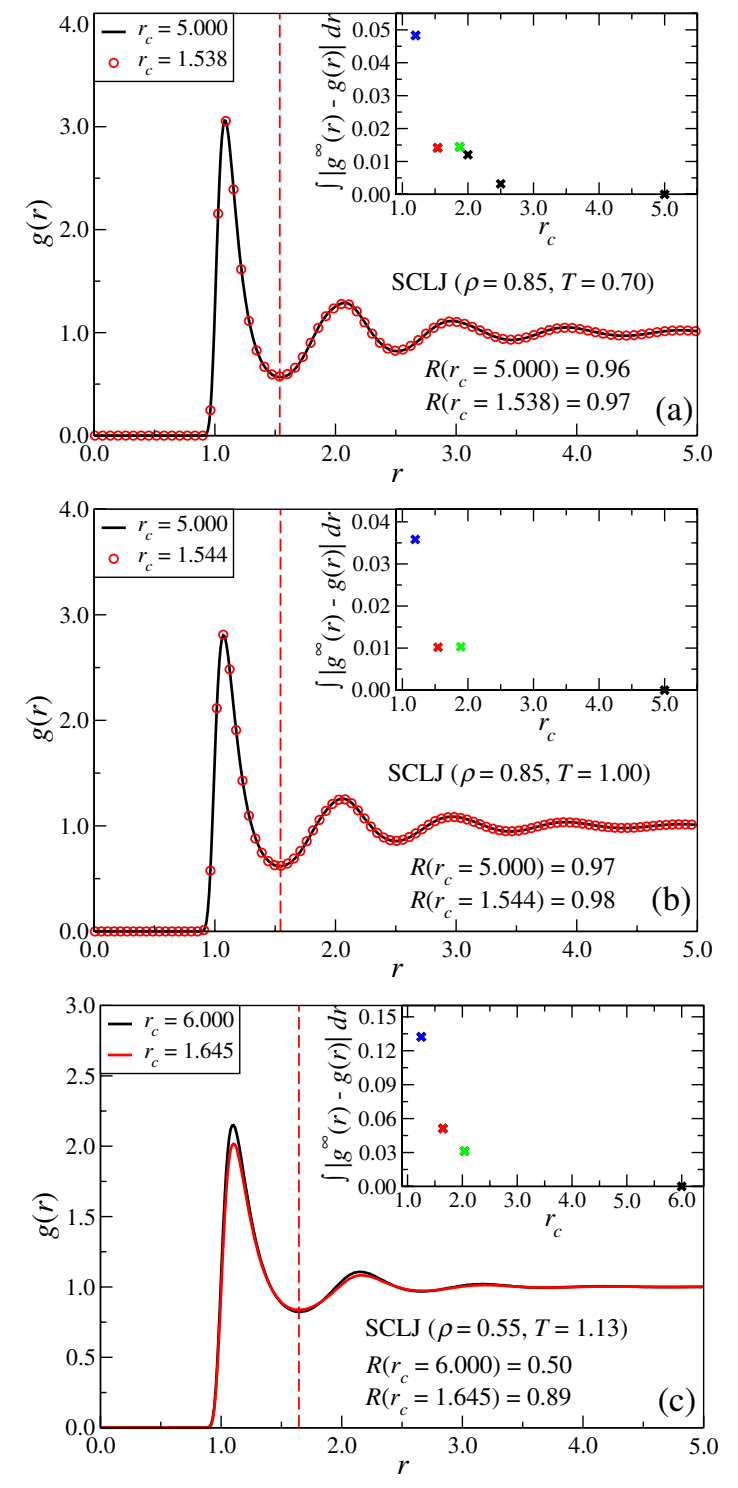

FIG. 4. RDFs for the SCLJ liquid at three different state points: (a) $\rho=0.85, T=0.70(R=0.96)$; (b) $\rho=0.85, T=1.00(R=$ $0.97)$; (c) $\rho=0.55, T=1.13(R=0.50)$. The black curves show reference simulations with large cutoffs; the red dots and curve show results from simulations with a FCS cutoff (marked by the vertical red dashed lines). The insets quantify the deviation in RDF from the reference RDF as functions of the cutoff. At all three state points, deviations increase significantly when the cutoff enters the FCS (blue crosses in the insets). For state points (a) and (b), which are strongly correlating $(R>0.9)$, a FCS cutoff leads to accurate results. This is not the case for state point (c).

simulations with a large cutoff (reference system). The insets quantify the deviations in the simulated RDF from the reference RDF as a function of the cutoff. The reference RDF of Figs. 4(a) and 4(b) is clearly well represented using a FCS cutoff, while choosing the cutoff inside the FCS results in significant deterioration. At low density [Fig. 4(c)], deviations occur between FCS-cutoff simulations and the reference system. As mentioned, the SCLJ liquid is strongly correlating in large parts of its phase 
diagram, but as density is lowered, the correlations decrease gradually and the liquid is no longer strongly correlating at state point (c), where $R=0.50$. These simulations suggest that only when a liquid is strongly correlating, is it possible to ignore interactions beyond the FCS.

Next, we investigated the SCLJ dynamics at the same three state points. The dynamics is studied via the incoherent ISF. The ISFs are shown in Fig. 5; at all state points the dynamics is well represented using a FCS cutoff.

We proceed to investigate mixtures of two different particles $(A$ and $B)$ interacting with LJ type potentials.
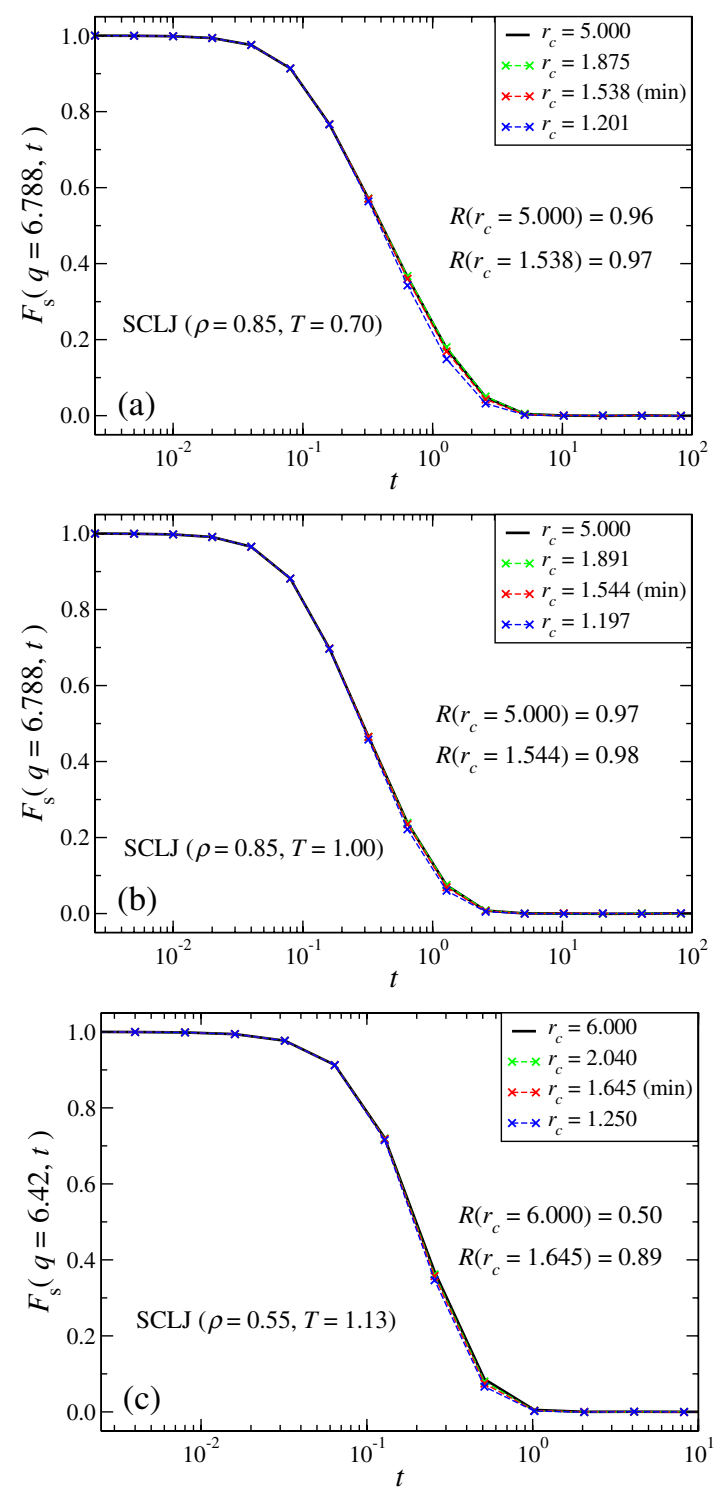

FIG. 5. Incoherent ISFs for the SCLJ liquid at the state points of Fig. 4. The black curves give the reference cutoff results, the red curves give the FCS-cutoff results, the blue curves give results for a cutoff at the half-height towards the first maximum of the RDF, and the green curves give results for a cutoff to the right of the minimum. (a) $\rho=0.85, T=0.70 \quad(R=0.96)$; (b) $\rho=0.85, T=1.00(R=0.97)$; (c) $\rho=0.55, T=1.13$ $(R=0.50)$.
The cutoff used for all three interactions $(A A, A B, B B)$ is placed at the same distance, referring to $\sigma_{A A}$. In Fig. 6 the reference and FCS-cutoff results are shown for the $A A$-particle RDFs of generalized KABLJ mixtures with repulsive exponent 12 and attractive exponents $n=4,10$. For all investigated state points a FCS cutoff gives accurate results. We found the same using the standard repulsive exponent $n=6$ (results not shown) [32].

The A-particle ISFs for the state points of Fig. 6 are shown in Fig. 7. For the KABLJ mixture also, placing the cutoff inside the FCS (blue curves) fails to reproduce the dynamics properly, whereas the dynamics is well approximated using a FCS cutoff (red). Slight deviations are noted for the red curves, an issue considered in Appendix B, which discusses alternatives for delimiting the FCS. Similar results are found for the $B$ particles (results not shown).

We also simulated the Wahnstrom 50/50 binary LJ mixture [48], finding again that whenever $R>0.9$, the structure and dynamics are well reproduced using a FCS cutoff. We do not show these results, but show instead results for the $A A$ coherent ISF at one state point (Fig. 8). Again, the FCS cutoff (red crosses) gives the correct
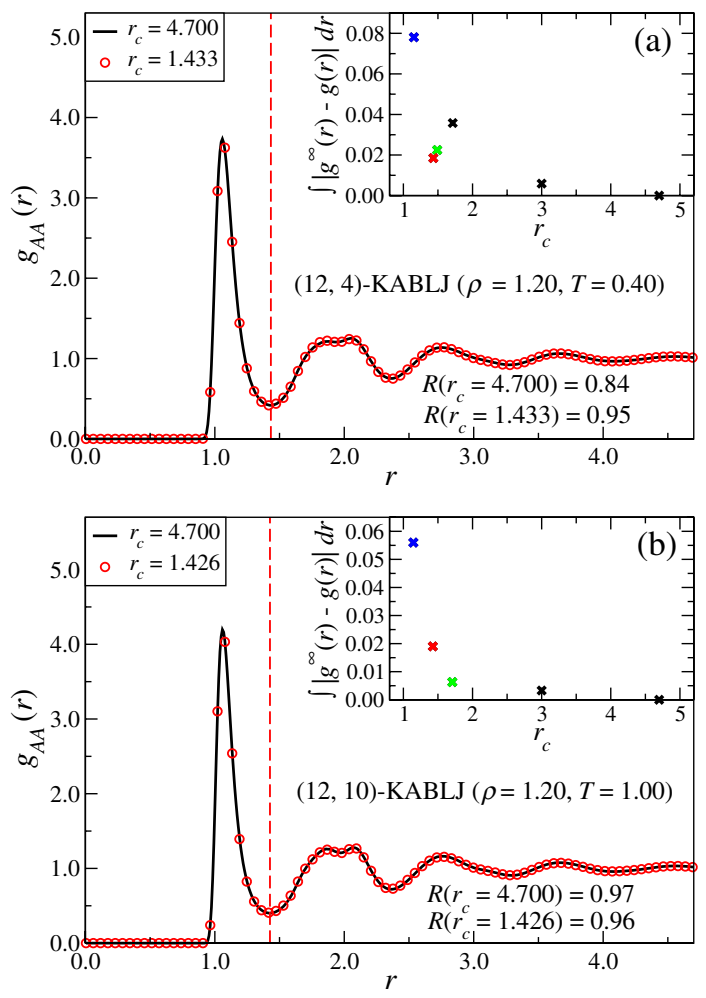

FIG. 6. RDFs for generalized KABLJ mixtures with repulsive exponent 12 and attractive exponents $n=4,10$. The black curves give the reference cutoff results; the red circles give the FCScutoff results. The insets quantify the deviation in RDF from the reference RDF as functions of the cutoff. (a) $n=4, \rho=1.20$, $T=0.40 \quad(R=0.84) ; \quad(b) \quad n=10, \quad \rho=1.20, \quad T=1.00$ $(R=0.97)$. 

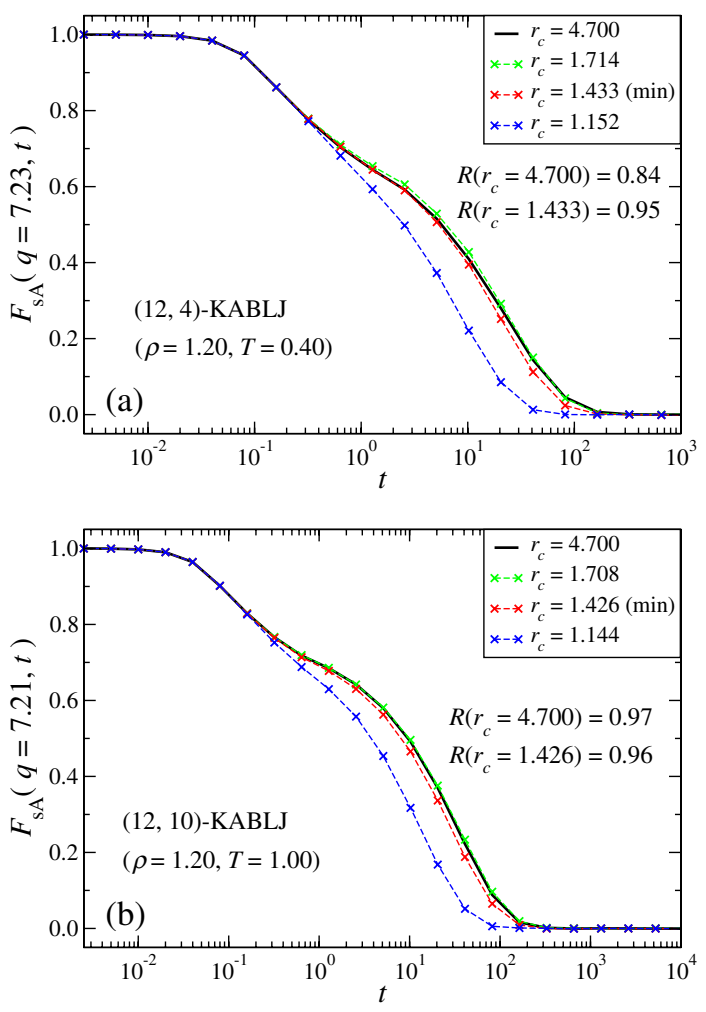

FIG. 7. Incoherent ISFs for generalized KABLJ mixtures with repulsive exponent 12 and attractive exponents $n=4,10$. The red and black curves give, respectively, results for FCS cutoffs and large reference cutoffs. (a) $n=4, \rho=1.20, T=0.40$ $(R=0.84)$; (b) $n=10, \rho=1.20, T=1.00$ ( $R=0.97)$.

dynamics, whereas reducing the cutoff further does not give proper results (blue crosses).

In summary, for all LJ-type systems, whenever there are strong virial potential-energy correlations $(R>0.9)$, a FCS cutoff gives accurate results for both the structure and dynamics.

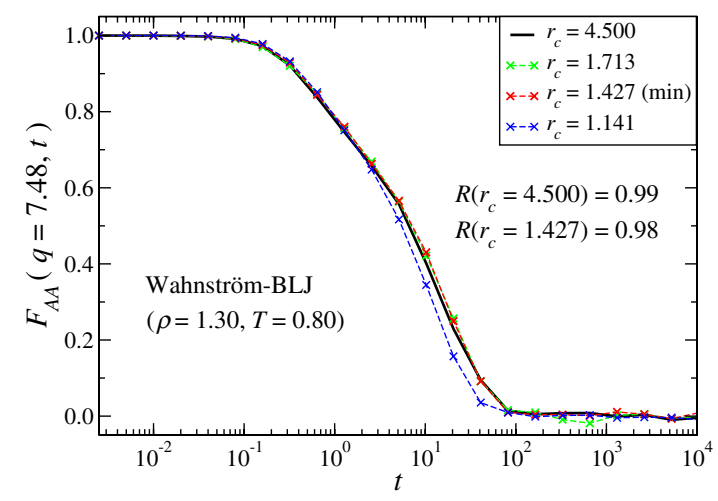

FIG. 8. Coherent ISF of the $A$ particles for the Wahnstrom $50 / 50$ binary LJ liquid at the wave vector defined by the static structure factor maximum. The red and black curves give, respectively, results for a FCS cutoff and large reference cutoff at the state point where $\rho=1.30$ and $T=0.80$.

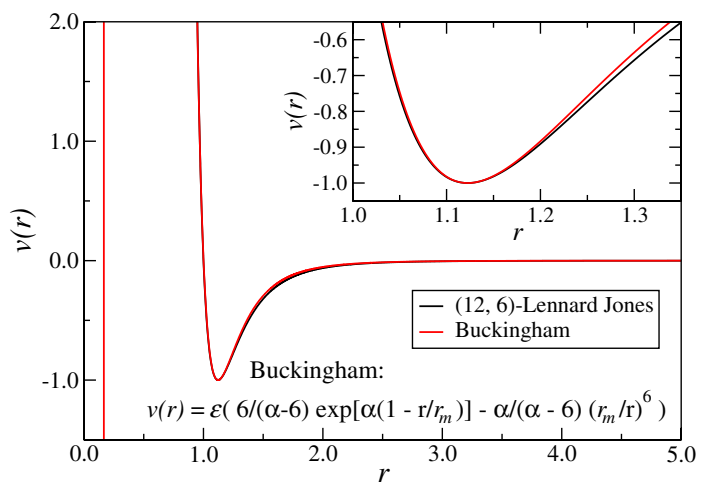

FIG. 9. The Buckingham pair potential (red) and the LJ pair potential (black). The parameters of the Buckingham potential were chosen such that the LJ potential is well approximated in the repulsive region.

\section{Buckingham liquid}

Next, we consider the single-component Buckingham liquid (SCB). The Buckingham potential $[49,50]$ is similar to the LJ potential, but does not have an IPL repulsive term; instead the potential's short-distance behavior follows a steep exponential (Fig. 9). Consequently, the Buckingham potential does not diverge at $r=0$. The parameters of the
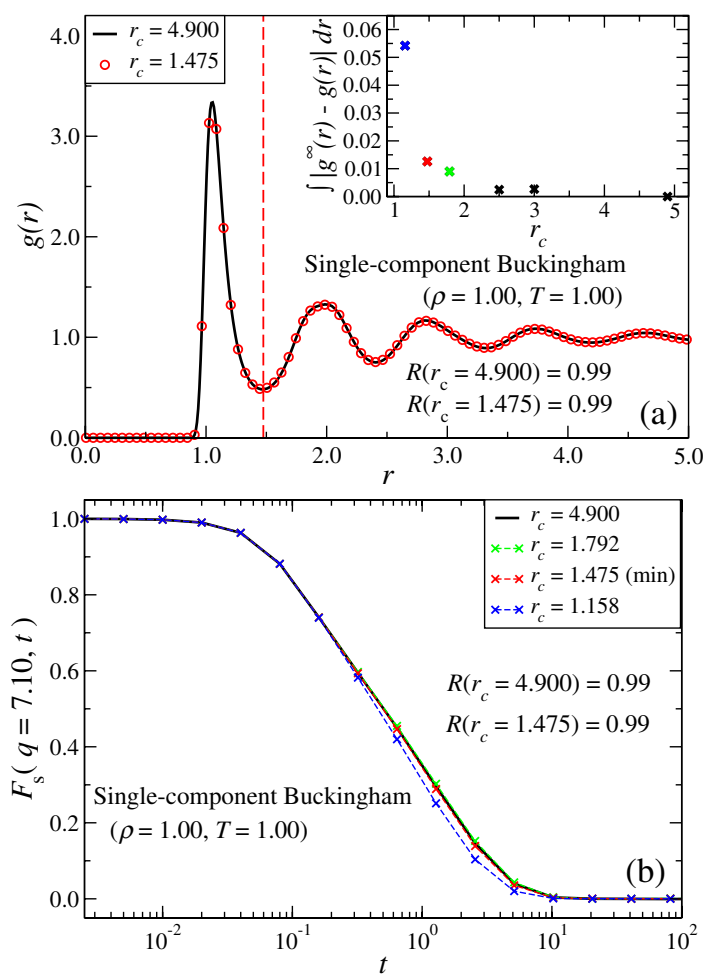

FIG. 10. The effect on the structure and dynamics of varying the cutoff for the SCB liquid. The red and black curves give, respectively, results for a FCS cutoff and a large reference cutoff. (a) RDF at $\rho=1.00$ and $T=1.00(R=0.99)$. The inset quantifies the deviation in RDF from the reference RDF as a function of the cutoff. (b) Incoherent ISF at the same state point. 


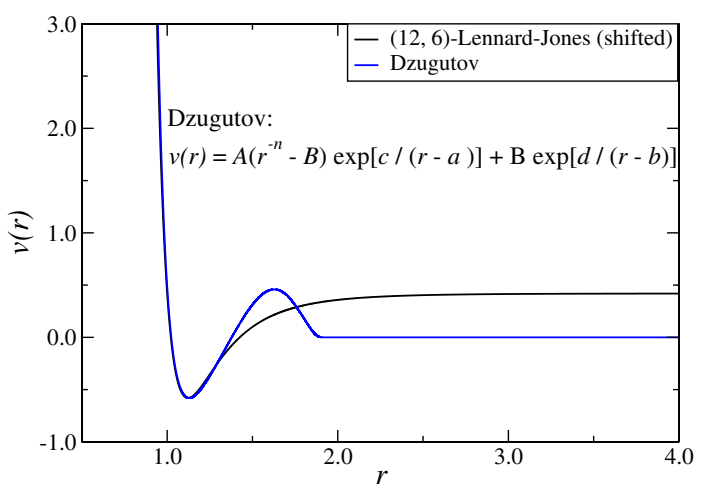

FIG. 11. The DZ pair potential [51] (blue curve). Also shown is the LJ pair potential (black curve). The DZ potential approximates the LJ potential around the first minimum, but has a maximum at larger distances.

Buckingham potential (Appendix A) were chosen such that the LJ potential is well approximated in the lower-energy repulsive part of the potential (Fig. 9).

Figures 10(a) and 10(b) show, respectively, the RDF and ISF for the SCB liquid. The SCB liquid is strongly correlating [50] and a FCS cutoff works well.

\section{Dzugutov liquid}

Figure 11 shows the Dzugutov (DZ) pair potential [51], which was originally suggested as a model potential for which crystallization is impeded by energetically punishing particle separations corresponding to the nextnearest-neighbor distance of crystallographic local order. At short distances the DZ pair potential approximates the LJ potential.

Figures 12(a) and 12(b) show, respectively, the RDF and the coherent ISF of the DZ system. For this system, the use of a FCS cutoff leads to poor results. This is not

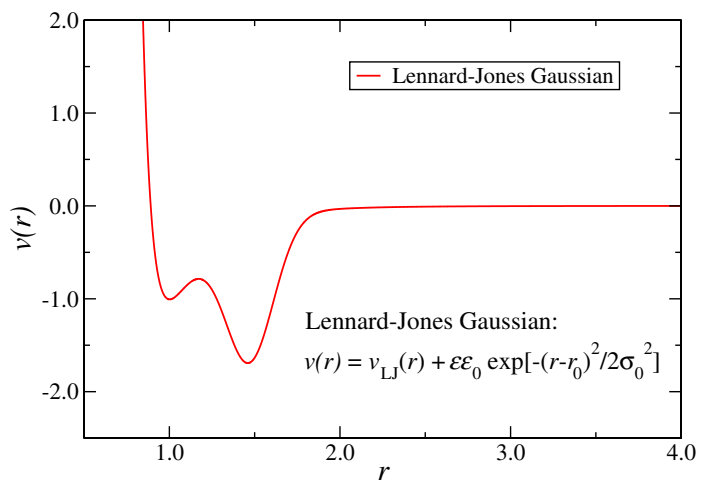

FIG. 13. The LJG pair potential [52] constructed by adding a Gaussian to the LJ potential. Two distinct minima are present.

surprising given the fact that using a FCS cutoff removes the maximum of the DZ potential. What is important here, however, is that the poor FCS-cutoff results correlate with the fairly weak virial potential-energy correlations $(R=0.71)$. This suggests studying other nonstrongly correlating liquids in order to investigate whether this is a general trend.

\section{E. Lennard-Jones Gaussian liquid}

The Lennard-Jones Gaussian (LJG) liquid [52] is a nonstrongly correlating liquid with the two-minimum pair potential shown in Fig. 13. The parameters of the LJG model (Appendix A) are such that the LJG potential minimum does not coincide with that of the SCLJ system [53].

Results from the simulating structure and dynamics of the LJG liquid are shown in Figs. 14(a) and 14(b). The FCS cutoff does not give the correct RDF. Deviations in the dynamics are fairly small, likely due to the fact that the dynamics is fast [compare, e.g., the time scale of the decay to that of the DZ liquid in Fig. 12(b)].
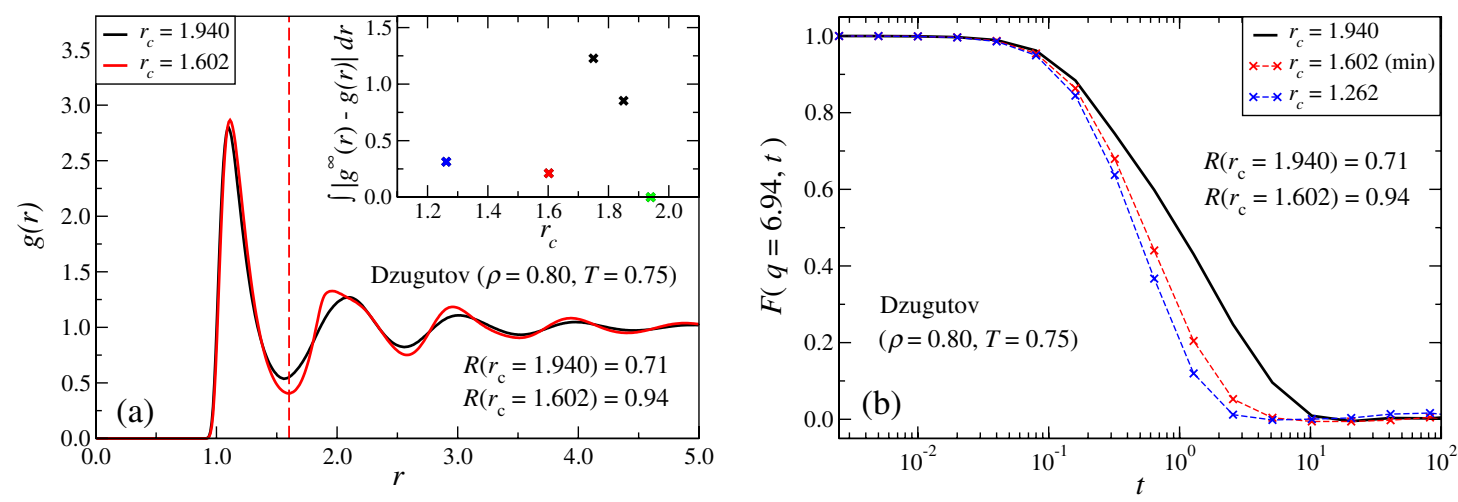

FIG. 12. The effect on the structure and dynamics of varying the cutoff for the DZ liquid at $\rho=0.80$ and $T=0.75(R=0.71)$. The red and black curves give, respectively, results for a FCS cutoff and a large reference cutoff. (a) RDF. The inset quantifies the deviation in RDF from the reference RDF as a function of the cutoff. (b) The coherent ISF at the same state point, including here results for a cutoff within the FCS (blue crosses). 

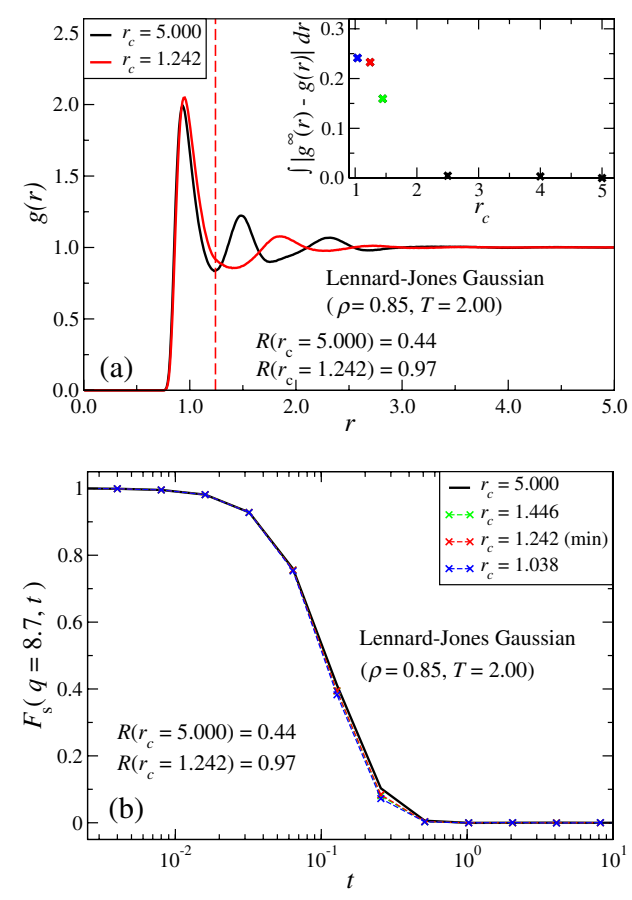

FIG. 14. The effect of the cutoff on the structure and dynamics for the LJG liquid. The red and black curves give, respectively, results for a FCS cutoff and a large reference cutoff. (a) RDF at $\rho=0.85$ and $T=2.00(R=0.44)$. The inset quantifies the deviation in RDF from the reference RDF (black curve) as a function of the cutoff. (b) Incoherent ISF at the same state point.
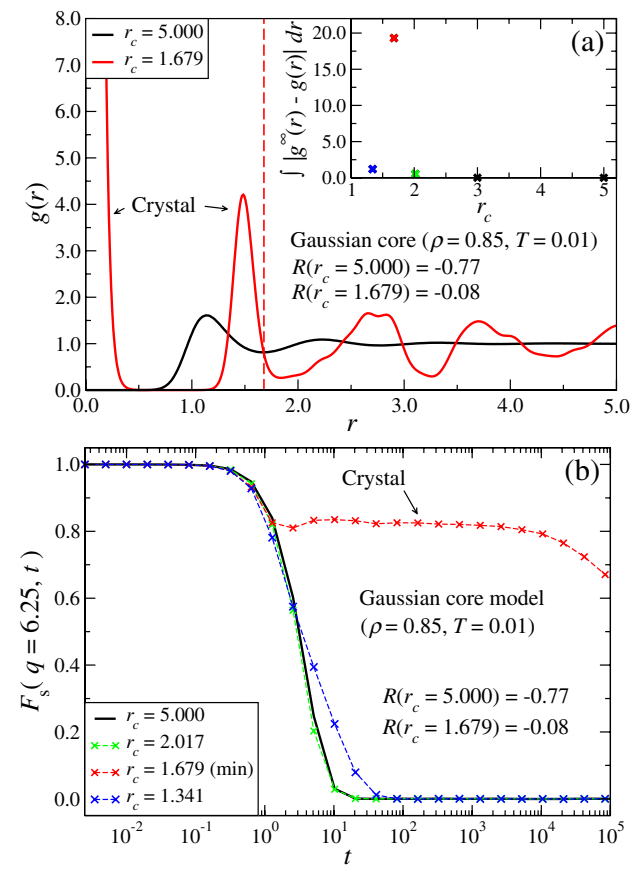

FIG. 15. The effect on the structure and dynamics of varying the cutoff for the GCM liquid. The red and black curves give, respectively, results for a FCS cutoff and a large reference cutoff. (a) RDF at $\rho=0.85$ and $T=0.01(R=-0.77)$. The inset quantifies the deviation in RDF from the reference RDF as a function of the cutoff. The red curve represents a crystallized state. (b) Incoherent ISF at the same state point.

\section{F. Gaussian core model}

The Gaussian core model (GCM) [54,55], which is not strongly correlating, is defined by a Gaussian pair potential and thus has a finite potential energy at zero separation. The high-density regime of the GCM model $(\rho>1.5)$ has recently received attention as a singlecomponent model glass former [56], because it is not prone to crystallization and shows the characteristic features of glass-forming liquids (large viscosity, two-step relaxation, etc.).

Figure 15 shows the RDF and ISF for the GCM liquid. The GCM crystallizes when a FCS cutoff is used. For this reason, obviously, a FCS cutoff is not able to reproduce the structure and dynamics of the reference system. Note, however, that crystallization does not occur when the cutoff is chosen in the neighborhood of the FCS cutoff (see the inset).

\section{G. The Hansen-McDonald molten salt model}

The final atomic system we studied is the so-called singly charged molten salt model proposed by Hansen and McDonald [57]. In Fig. 16 we see that the structure
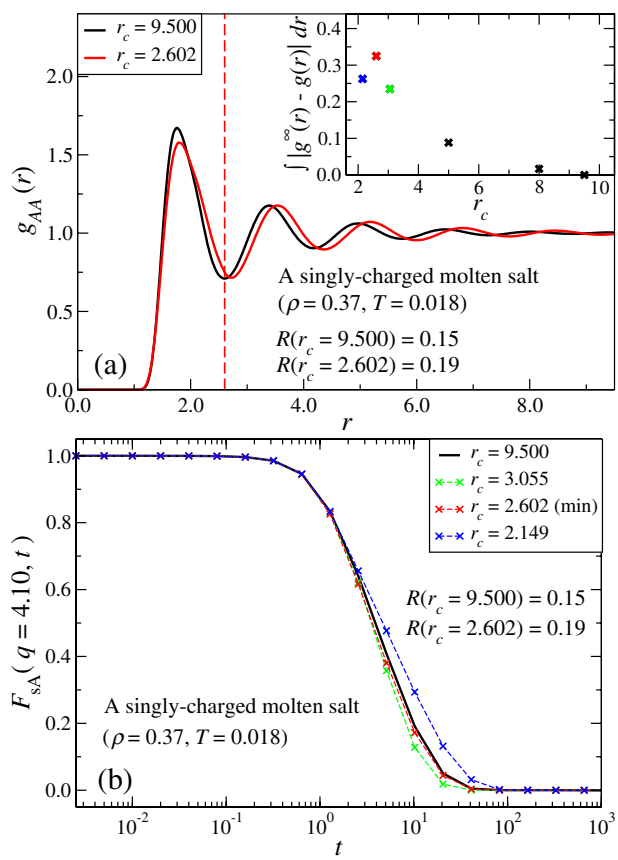

FIG. 16. The effect on the structure and dynamics of varying the cutoff for the Hansen-McDonald single-charged molten salt model. Because of the competing interactions (Coulomb and $n=9$ repulsive IPL) this model is not strongly correlating. The red and black curves give, respectively, results for a FCS cutoff and a large reference cutoff. (a) $A A$-particle RDF at $\rho=0.37$ and $T=0.018(R=0.15)$. The inset quantifies the deviation in RDF from the reference RDF as a function of the cutoff. (b) $A$-particle incoherent ISF at the same state point. 
is not represented well by a FCS cutoff. Interestingly, the dynamics is well reproduced using this cutoff-even better, in fact, than for a larger cutoff [Fig. 16(b), green curve].

\section{H. Two strongly correlating molecular model liquids}

We finish the presentation of the numerical results by giving data for three molecular model liquids. In this subsection, data are given for two strongly correlating molecular liquid models, the Lewis-Wahnstrom orthoterphenyl (OTP) model $[58,59]$ and the asymmetric dumbbell model [19], which represent a molecule by three and two rigidly bonded LJ spheres, respectively. The next subsection gives data for a rigid water model.

Figures 17(a) and 17(b) show the LJ particle RDF and ISF of the OTP model. Both quantities are well approximated using a FCS cutoff, although slight deviations are noted for the ISF (red curve, see Appendix B for considerations concerning this). The OTP model is a borderline strongly correlating liquid $(R=0.91)$.
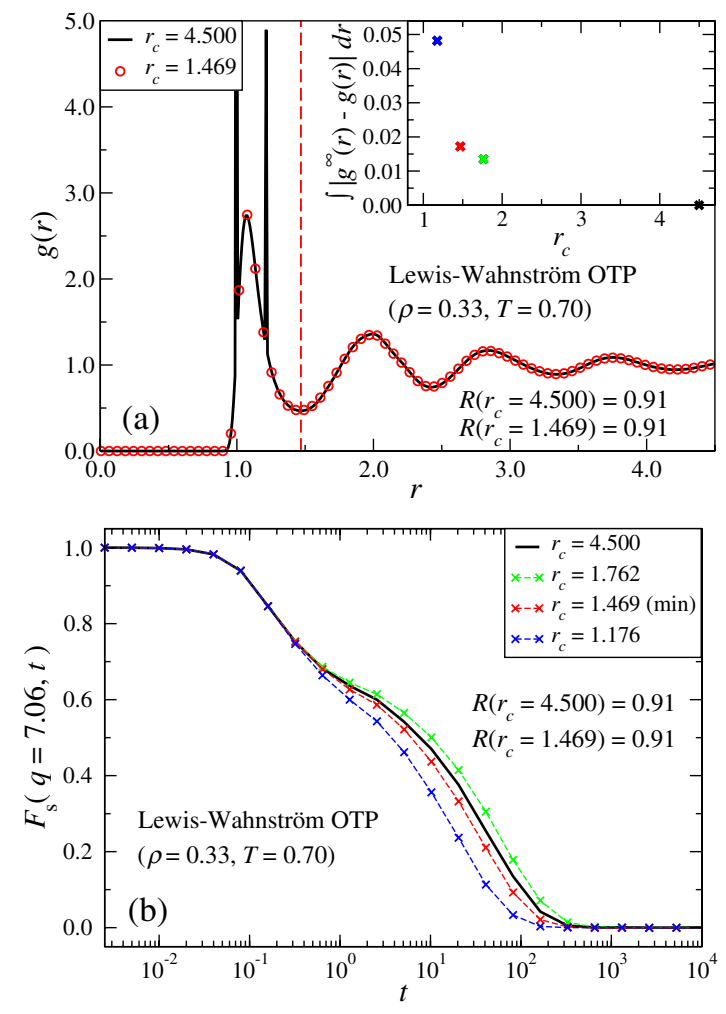

FIG. 17. The effect on the structure and dynamics of varying the cutoff for the Lewis-Wahnstrom OTP model. The red and black curves give, respectively, results for a FCS cutoff and a large reference cutoff. (a) RDF of the LJ particles at $\rho=0.33$ and $T=0.70(R=0.91)$. The inset quantifies the deviation in RDF from the reference RDF as a function of the cutoff. The spikes derive from the bonds. (b) Incoherent ISF at the same state point.
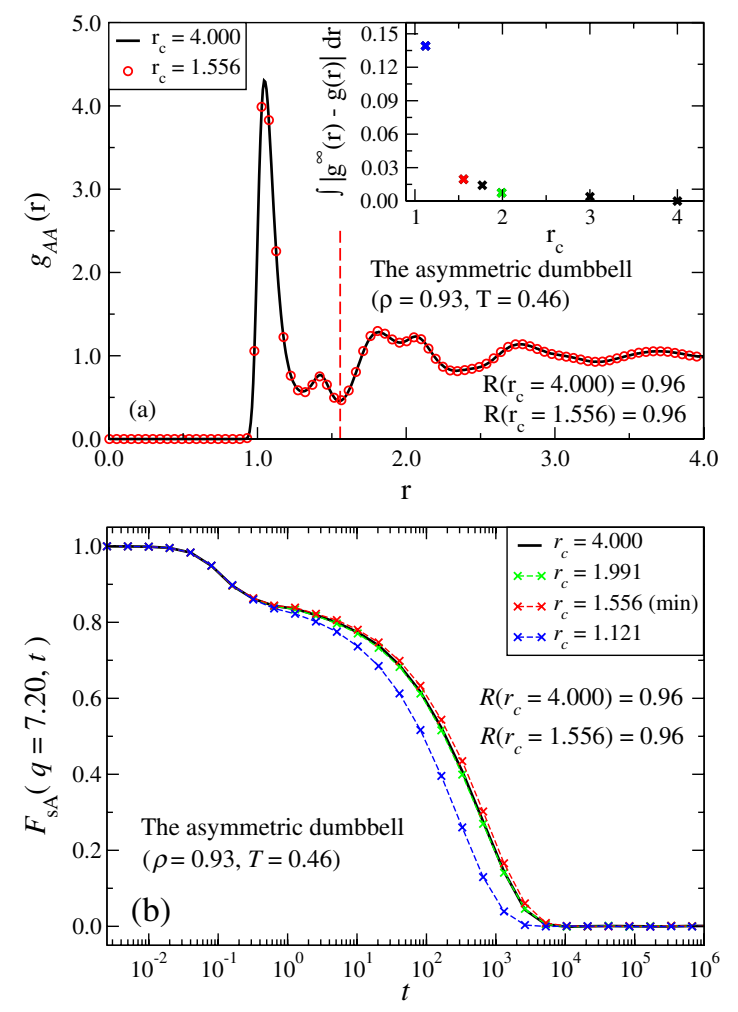

FIG. 18. The effect on the structure and dynamics of varying the cutoff for the asymmetric dumbbell model. The red and black curves give, respectively, results for a FCS cutoff and a large reference cutoff. Note that in this case the FCS cutoff is defined by using the second minimum (the first minimum is not the absolute minimum). (a) $\mathrm{RDF}$ at $\rho=0.93$ and $T=0.46(R=$ 0.96). The inset quantifies the deviation in RDF from the reference RDF (black curve) as a function of the cutoff. (b) A-particle incoherent ISF at the same state point. For the lowest cutoff the system crystallized.

Figures 18(a) and 18(b) show corresponding figures for the large $(A)$ particle of the asymmetric dumbbell model at a viscous state point. The use of a FCS cutoff gives accurate results for both the structure and dynamics. The FCS cutoff was placed at the second minimum of the $A A \mathrm{RDF}$, because the $A A \mathrm{RDF}$ has here a lower value than at the first minimum. If the cutoff is placed at the first minimum, clear deviations are found (data not shown).

\section{Rigid SPC/E water model}

We consider finally the rigid single-point charge (SPC/ E) water model [60] (Fig. 19). This model is not strongly correlating at ambient conditions, a fact that directly reflects water's well-known density maximum [18]. The structure of the SPC/E water model is not well represented using a FCS cutoff. Interestingly, the FCS-cutoff dynamics shows only slight deviations from that of the reference curve (black). 

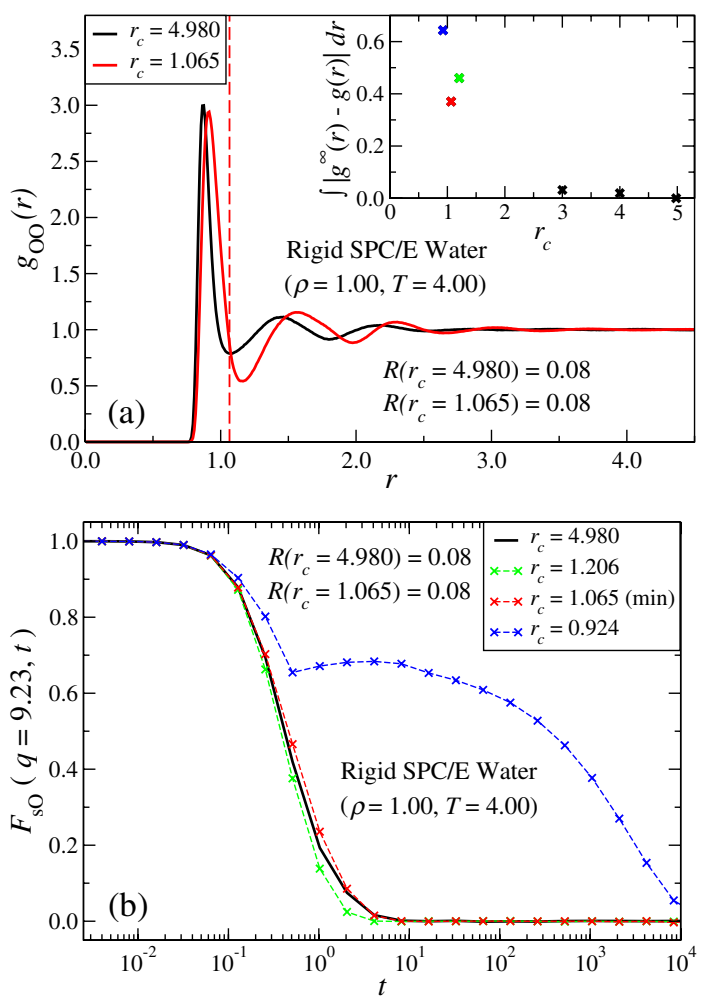

FIG. 19. The effect on the structure and dynamics of varying the cutoff for the rigid SPC/E water model [60]. The red and black curves give, respectively, results for a FCS cutoff and a large reference cutoff. (a) Oxygen-oxygen RDF at $\rho=1.00$ and $T=4.00(R=0.08)$. The inset quantifies the deviation in RDF from the reference RDF as a function of the cutoff. (b) Oxygen incoherent ISF at the same state point.

\section{SUMMARIZING THE SIMULATION RESULTS}

The previous section showed that the structure and dynamics are well approximated in simulations using a FCS cutoff for the following atomic and molecular systems:

(1) Inverse power-law systems $(n=18,6,4)$,

(2) single-component Lennard-Jones liquid at density $\rho=0.85$,

(3) generalized Kob-Andersen binary Lennard-Jones mixtures,

(4) Wahnstrom binary Lennard-Jones mixture,

(5) single-component Buckingham liquid,

(6) Lewis-Wahnstrom OTP model,

(7) asymmetric dumbbell model.

These systems are all strongly correlating [17,18,20,21,24]. Thus, for strongly correlating liquids, it is enough to know the intermolecular interactions within the FCS in order to accurately simulate the structure and dynamics.

The simulations showed further that for all of the following atomic and molecular systems, structure and/or dynamics are not properly reproduced when a FCS cutoff is used:
(1) single-component Lennard-Jones liquid at density $\rho=0.55$,

(2) Dzugutov liquid,

(3) Lennard-Jones Gaussian liquid,

(4) Gaussian core model,

(5) Hansen-McDonald molten salt model,

(6) rigid SPC/E water model.

For all these systems, larger cutoffs are needed in order to faithfully reproduce the system's physics. None of the latter liquids are strongly correlating.

In conclusion, a shifted-forces FCS cutoff leads to accurate results if and only if the liquid is strongly correlating at the state point in question. We know of no exceptions to this empirical rule. This suggests that strongly correlating liquids are characterized by the property that intermolecular interactions beyond the FCS can be safely ignored.

\section{THE REAL ESSENCE OF SIMPLICITY OF LIQUIDS}

As discussed in the Introduction, a definition of simple liquids is most useful if it identifies their real essence in Locke's understanding of the term [3], the underlying fundamental characteristic from which these liquids' simple features, their nominal essences, follow. We suggest below that the class of simple liquids is to be identified with the class of strongly correlating liquids (Sec. IV C). This is motivated by first summarizing the many simple properties of strongly correlating liquids (Sec. IVA), then showing that this class of liquids can be characterized from three different perspectives: mathematically, physically, and chemically (Sec. IV B). This gives three very different characterizations, indicating that the class of strongly correlating liquids is fundamental, and further motivating the suggestion that the real essence of liquid simplicity is the existence of strong correlations of virial potential-energy equilibrium $N V T$ fluctuations. By connecting to the chemists' concept of nonassociated liquids, we then discuss which real-world liquids are simple (Sec. IV D), liquids near interfaces (Sec. IV E), and give examples of complex liquid properties (Sec. IV F). Finally, Sec. IV G points out that our results call into question the physical basis of traditional perturbation theory, which assumes quite different roles of the attractive and the repulsive forces; this distinction is not deep and fundamental for simple liquids.

\section{A. Strongly correlating liquids and their properties}

The simple properties of strongly correlating liquids follow from the existence of "isomorphs" in their phase diagram (see below). Some simple properties were identified before isomorphs were defined in 2009 [21], however, for instance that

(1) all eight fundamental thermoviscoelastic response functions are given in terms of just one, i.e., the dynamic Prigogine-Defay ratio is close to unity [16], 
(2) aging may be described in terms of merely one extra parameter $[18,20]$,

(3) power-law density scaling [61] is obeyed to a good approximation; i.e., for varying density and temperature, the relaxation time is a function of $\rho^{\gamma / T}[19]$.

An isomorph is an equivalence class of state points in the phase diagram. Two state points $\left(\rho_{1}, T_{1}\right)$ and $\left(\rho_{2}, T_{2}\right)$ are defined to be isomorphic [21] if the following holds: Whenever one microconfiguration of state point (1) and one of state point (2) have the same reduced coordinates [i.e., $\rho_{1}^{1 / 3} \mathbf{r}_{i}^{(1)}=\rho_{2}^{1 / 3} r_{i}^{(2)}$ for all particles $i$ ], these two microconfigurations have proportional configurational Boltzmann factors,

$$
e^{-U\left(\mathbf{r}_{1}^{(1)}, \ldots, \mathbf{r}_{N}^{(1)}\right) / k_{B} T_{1}}=C_{12} e^{-U\left(\mathbf{r}_{1}^{(2)}, \ldots, \mathbf{r}_{N}^{(2)}\right) / k_{B} T_{2}}
$$

For most systems, the isomorph concept is approximate just as $W U$ correlations are rarely perfect. Thus, we do not require Eq. (5) to be rigorously obeyed for all microconfigurations, but only to a good approximation for all physically relevant microconfigurations. By this we mean microconfigurations that are not a priori unimportant for the physics. An isomorph defines a continuous curve of state points in the liquid's phase diagram.

Appendix A of Ref. [21] showed that a liquid is strongly correlating if and only if it has isomorphs to a good approximation. This was confirmed in Refs. [21,24], which showed that Lennard-Jones-type atomic liquids have good isomorphs. Likewise, Ref. [62] recently showed that the strongly correlating Lewis-Wahnstrom OTP and asymmetric dumbbell models have good isomorphs.

Equation (5) has many consequences. These were derived and discussed in detail in the original isomorph paper from 2009 (Ref. [21]), to which the reader is referred. Basically, the structure and dynamics at two isomorphic state points are identical in reduced units. Quantities that are invariant along an isomorph include (but are not limited to)

(1) The excess entropy, i.e., the entropy in excess of the ideal-gas entropy at the same density and temperature- this is the configurational contribution to the entropy (a quantity that is negative because a liquid is always more ordered than an ideal gas at same density and temperature).

(2) All $N$-body entropy terms. Recall that the excess entropy can be expanded in a series of two-body, three-body, etc., terms; each term is invariant along an isomorph [21].

(3) The isochoric heat capacity.

(4) The structure in reduced units (defined by $\tilde{\mathbf{r}}_{i} \equiv$ $\rho^{1 / 3} \mathbf{r}_{i}$ for all particles $i$ ). Not only the radial distribution function, but all higher-order distribution functions are isomorph invariant in reduced units.

(5) The Newtonian $N V E$ and Nosé-Hoover $N V T$ equations of motion in reduced units; likewise Brownian dynamics.
(6) All autocorrelation functions in reduced units.

(7) All average relaxation times in reduced units.

(8) Reduced transport coefficients such as the diffusion coefficient, viscosity, etc.

Isomorphs have the further interesting property that there is no relaxation for an instantaneous change of temperature and density when jumping from an equilibrated state point to a different state point isomorphic with the initial state. The absence of relaxation derives from the fact that the Boltzmann probabilities of scaled microconfigurations are identical. Such "isomorph jumps" have been shown to work very well for the KABLJ liquid [21], for the asymmetric dumbbell and for the Lewis-Wahnstrom OTP molecular models [62]. Moreover, the effective temperature of a glass prepared by a temperature-density jump from an equilibrium state of a strongly correlating liquid depends only on the final density [22]; this provides yet another example of a simple feature of these liquids.

Some further predictions for the class of strongly correlating liquids deriving from the existence of isomorphs are

(1) The solid-liquid coexistence curve is an isomorph [21,24]. This implies invariance along the coexistence curve of the reduced structure factor, the reduced viscosity, the reduced diffusion constant, etc., as well as pressure invariance of the melting entropy and the reduced-unit Lindemann melting criterion [21].

(2) Collapse of the two-order-parameter maps of Debenedetti et al. [63-67] to one-dimensional curves [21].

(3) Isochronal superposition [68], i.e., the fact that when pressure and temperature are varied, the average relaxation time determines the entire relaxation spectrum [21].

The above listed properties of strongly correlating liquids all reflect one or the other kind of simplicity of strongly correlating liquids. A final, recently established simple property is a thermodynamic separation identity: For all strongly correlating liquids, if $s$ is the excess entropy per particle, the temperature as a function of $s$ and density $\rho$ factorizes as follows [69]:

$$
T=f(s) h(\rho) .
$$

Equation (6) has a number of consequences [69], for instance, the configurational Gruneisen equation of state and that the isomorphs of LJ liquids-in particular, the LJ solid-liquid coexistence curve-are given by $\left(A \rho^{4}-B \rho^{2}\right) / T=$ const $[70,71]$.

\section{B. Mathematical, physical, and chemical characterization of strongly correlating liquids}

At a given state point, if the average potential energy is denoted by $\langle U\rangle$, the constant-potential-energy 
hypersurface is defined by $\Omega=\left\{\left(\mathbf{r}_{1}, \ldots, \mathbf{r}_{N}\right) \in\right.$ $\left.R^{3 N} \mid U\left(\mathbf{r}_{1}, \ldots, \mathbf{r}_{N}\right)=\langle U\rangle\right\}$. This is a compact, Riemannian $(3 N-1)$-dimensional differentiable manifold. Each state point has its own such hypersurface. In this way, a family of high-dimensional manifolds is defined throughout the phase diagram. In Appendix A of Ref. [21] it was shown that the reduced-unit constantpotential-energy manifold is invariant along a strongly correlating liquid's isomorphs, and that, conversely, invariance curves exist for these manifolds only for strongly correlating liquids. Thus, for such liquids, these manifolds constitute a one-parameter family of manifolds, not two-parameter families as expected from the fact that the phase diagram is two-dimensional. This provides a mathematical characterization of the class of strongly correlating liquids.

The physical characterization of this class was discussed already: A liquid is strongly correlating if and only if it has isomorphs to a good approximation; this is shown in Appendix A of Ref. [21]. The proof utilizes that a liquid is strongly correlating if and only if its constant-virial hypersurfaces in the $3 \mathrm{~N}$-dimensional configuration space are (almost) identical to its constant-potential-energy hypersurfaces.

The chemical characterization of strongly correlating liquids is the property documented in the present paper: A liquid is strongly correlating at a given state point if and only if the liquid's structure and dynamics are accurately calculated by simulations that ignore interactions beyond the first coordination shell. This is an empirical finding for which we have, at present, no compelling argument. How can one justify this FCS characterization of strongly correlating liquids? Note first that the property of insignificance of interactions beyond the FCS is an isomorph invariant: If a liquid has good isomorphs and if a FCS cutoff works well at one state point, FCS cutoffs must work well for all its isomorphic state points. Thus, the chemical characterization of strongly correlating liquids is consistent with the fact that these liquids have isomorphs. Note further that it has been shown for the Lennard-Jones liquid that almost all of the fluctuations in virial and potential energy come from interparticle separations within the FCS [18]. Finally, we give an nonrigorous argument that a SF cutoff works well for any strongly correlating liquid: Consider an atomic liquid with pair interaction $v(r)$ that is strongly correlating at the state point in question. Since virial and potential-energy fluctuations correlate, one can replace $v(r)$ by $\lambda r v^{\prime}(r)$ where $\lambda$ is some constant. Thus the radial force $f(r)=-v^{\prime}(r)$ can be replaced by $-\lambda\left[r v^{\prime}(r)\right]^{\prime}=\lambda\left[f(r)+r f^{\prime}(r)\right]$. This implies for some constant $\mu$ that $f(r) \sim \mu r f^{\prime}(r)$ where $\sim$ indicates equivalence in MD simulations. Assuming the SF cutoff system is likewise strongly correlating, we get $f_{\mathrm{SF}}(r) \sim \mu r f_{\mathrm{SF}}^{\prime}(r)$. Since $f^{\prime}(r)=f_{\mathrm{SF}}^{\prime}(r)$, one concludes that $f(r) \sim f_{\mathrm{SF}}(r)$.
Most likely it is the existence of a well-defined FCS that implies the almost cancellation of the linear term of the shifted-force potential. The fact that interactions beyond the FCS may be ignored shows that interactions are effectively short ranged, which means that the structure is dominated by what may be termed packing effects.

\section{Defining the class of simple liquids}

Section IVA listed several simple properties of strongly correlating liquids. Section IV B showed that this liquid class may be characterized from three quite different perspectives. It appears that the class of strongly correlating liquids is fundamental. Since the properties of strongly correlating liquids are generally simpler than those of liquids in general, we now propose the following definition: Simple liquid=strongly correlating liquid. This is the basic message of the present paper, which implies a quantification of the degree of simplicity via the number $R$ of Eq. (2), the $N V T$ ensemble equilibrium virial potentialenergy correlation coefficient.

Compared to the standard definition of simple liquids as those with radially symmetric pair interactions, there are some notable differences:

(1) Simplicity is quantified by a continuous variable, it is not an on/off property.

(2) The degree of simplicity generally varies throughout the phase diagram. Consequently, simplicity is not merely encoded in a liquid's intermolecular interactions. In fact, most strongly correlating liquids lose this property as density is lowered and the gas phase is approached.

(3) Not all "atomic" liquids (i.e., with radially symmetric pair interactions) have simple regions in the low-pressure part of the phase diagram (compare the Dzugutov, Lennard-Jones Gaussian, Gaussian core, and molten salt models);

(4) Not all simple liquids are atomic (compare the Wahnstrom OTP and the asymmetric dumbbell models).

According to the new definition of liquid simplicity, the case where the potential energy is an Euler homogeneous function of the particle positions $(R=1)$ sets the gold standard for simplicity. This is consistent with the many simple properties of these liquids. Usually one has in mind IPL systems with pair interactions that scale with the distance as $r^{-n}$. However, $R=1$ systems as mentioned also include some with angular dependencies in the potential energy, as long as these scale with distance the same way as all other interactions. Because of the absence of attractions, IPL fluids have no liquid-gas phase transition. In this sense it may seem strange to claim that IPL fluids are the simplest liquids. However, more realistic strongly correlating liquids like the LJ liquid cease to be so when the liquid-vapor coexistence line is approached, showing that this phase transition cannot be understood in the framework of simple 
liquids. This contrasts with the liquid-solid phase transition, where, for instance, the fact that the coexistence line for simple liquids is an isomorph-confirmed for the LJ liquid [24] — explains several previously noted regularities [21].

Is the hard-sphere fluid simple? One may define a configurational virial function for this system, but it is not obvious how to define a potential-energy function that is different from zero. Thus, there is no meaningful correlation coefficient $R$ for hard-sphere fluids. On the other hand, the hard-sphere liquid may be regarded as the $n \rightarrow \infty$ limit of an IPL liquid, and it is well known that, for instance, the hard-sphere radial distribution function is close to that of, e.g., an $r^{-20}$ IPL liquid at a suitably chosen temperature. This would indicate that hard-sphere liquids are simple, which is consistent with the prevailing point of view. Another interesting case is that of the WCA version of the LJ liquid, which cuts off all attractions by putting the force equal to zero beyond the potential-energy minimum. This liquid is strongly correlating [72]. Despite this, we found in simulations that the WCALJ liquid has somewhat poorer isomorphs than the LJ liquid.

It is possible that the hard-sphere liquid and the WCALJ liquid should be both excluded from the class of simple liquids on the grounds that their potentials are not analytic. For systems interacting via pair potentials, it could make good sense to add the extra requirement that the pair potential is an analytical function of the inverse pair distance, i.e., that an expansion exists of the form $v(r)=$ $\sum_{n} v_{n} r^{-n}$. Such an extra analyticity requirement would not exclude any strongly correlating liquids occurring in nature where all potentials are expected to be analytic.

\section{Which liquids in the real world are simple?}

Real-world liquids may be classified according to the nature of the chemical bonds between the molecules. There are five types of bonds [73], which are listed below with a few typical examples (polymeric systems may be added as a separate class):

(1) Van der Waals bonds (e.g., argon, toluene, butane, etc.);

(2) Metallic bonds (e.g., gold, aluminum, alloys, etc.);

(3) Hydrogen bonds (e.g., water, glycerol, ethanol, etc.);

(4) Ionic bonds (e.g., molten sodium chloride, molten potassium nitrate, room-temperature ionic liquids, etc.);

(5) Covalent bonds (e.g., silica and borate melts, etc.).

Most liquids involve elements of more than one type of chemical bond. For instance, van der Waals forces are present in all liquids; the first class consists merely of those liquids that only have van der Waals forces. Another borderline example is a dipolar organic liquid like di-butylphthalate, where van der Waals as well as Coulomb forces are present; the hydrogen-bonded liquid glycerol also has strong dipolar interactions, i.e., an element of the ionic bonds, etc.

Based on computer simulations and known properties of liquids, we believe that most or all van der Waals and metallic liquids are strongly correlating [14,16,18], i.e., simple. Liquids that are not simple are the hydrogen, ionically, and covalently bonding liquids. In these cases, the virial potential-energy correlations are weakened by the existence of competing interactions, either with different $r$ dependences (the ionically bonding liquids) or because angular and radial forces have different $r$ scaling (the hydrogen and covalently bonded liquids).

Metals play a special role as simple liquids, because their interatomic forces derive from collective interactions between ion cores and free electrons [8]. The resulting interaction is a nondirectional interaction between symmetric ion cores, i.e., these systems are simple in the traditional sense. Preliminary computer simulations show that metals are strongly correlating [17], so metals are also simple in the sense of the present paper. However, not all isomorph invariants are expected to apply for metals. For instance, the electron gas can influence the collective dynamics without any structural and relaxational counterpart [74,75], so isomorph invariance most likely breaks down for these (fast) collective degrees of freedom.

It should be emphasized that the above considerations refer to ambient or moderate pressure conditions. It was recently suggested that all liquids become strongly correlating at high pressure [76]. Thus, e.g., the molten silicates of the Earth's upper mantle are predicted to be simpler than molten silicates at ambient pressure.

\section{E. Liquids near interfaces}

It is interesting to consider liquids under more general circumstances, for instance under confinement or generally near interfaces. Liquids near interfaces show rich and complicated behavior. For instance, a liquid confined to the nanoscale may change its dynamic properties several orders of magnitude compared to the bulk system. Predicting these changes is an important challenge relevant for biological systems, engineered devices, etc. Recently, it was shown that some liquids retain bulk liquid behavior in confinement [77-80]. More specifically, it was shown that Rosenfeld's excess entropy scaling in the bulk persists in confinement and is, to a good approximation, independent of the wall-fluid interaction strength. This was shown for LJ and hard-sphere liquids, suggesting the possibility of extending the concept of a simple liquid beyond bulk systems. More work is needed, however, to clarify the relevance and consequences of the present definition of liquid simplicity near interfaces $[81,82]$.

\section{F. A note on complex liquid behavior}

Here we give a brief example showing that liquids, which are not simple in the above defined sense, often 
have complex properties [65,83-86]. Water with its correlation coefficient close to zero at ambient conditions is a prime example of a complex liquid. It is well known for water that a certain region of state points in the densitytemperature phase diagram exhibits anomalous thermodynamic behavior in the sense that isobaric heating implies densification. Numerical evidence indicates that these state points lie within a larger region with a diffusion anomaly, i.e., an increased diffusivity upon isothermal compression [65], a region that, in turn, lies within a larger region of a structural anomaly characterized by decreasing order upon isothermal compression [65].

Different order parameters exist for characterizing the structural order of liquids, some of which relate purely to an integral over the RDF [83-85]. In this way, it is possible to calculate the contribution to structural anomalies from the different coordination shells [83-85]. It has been shown $[84,85]$ that the structural anomaly of water and waterlike liquids is not a "first-shell effect. Rather, they reflect how structuring in second and more distant coordination shells responds to changes in thermodynamic or system parameters" $[84,85]$. Thus, the anomalous behavior of water derives from interactions beyond the FCS $[83,85]$. This is consistent with the results presented in this paper-water is not simple-since the structure and dynamics of strongly correlating liquids are given exclusively by the interactions within the FCS.

\section{G. To which extent do the assumptions of standard pertubation theory hold?}

The finding that the FCS plays a crucial role for a large class of systems may be taken as a modern demonstration of the classic van der Waals picture of liquids, in the sense that such liquids can be understood in terms of packing effects [87]. On the other hand, our results call into question the basis of traditional perturbation theory, which is conceptually also usually traced back to van der Waals [88]. Perturbation theory is based on the assumption of entirely different roles being played by the repulsive and the attractive forces [7,30,81,87-90]: The repulsive forces largely determine the structure and reduce the entropy compared to that of an ideal gas at same density and temperature; the attractive forces reduce the pressure and energy compared to that of an ideal gas. From the findings of this and a previous paper [32] it is clear, however, that this picture applies only at such low pressures that the FCS coincides with the region around the pair-potential minimum. At high pressure, the entire FCS is within the range of the repulsive forces; here, the attractive forces play little role for simple liquids. In general, what is important for a strongly correlating liquid is to take into account properly all forces from particles within the FCS - and only these. Thus, the well-known WCA reference system, which ignores the attractions, is a good reference only at such high pressure that all forces from particles within the FCS are repulsive [26,27,32].

The dominance of the FCS for simple liquids reflects the fundamental physics that the characteristic length defining the pair-potential minimum (e.g., $\sigma$ of the LJ potential) is much less important than generally believed: $\sigma$ determines the density of the low-pressure-condensed phase, but for simple liquids that is all. The physically relevant length for these liquids is the one given by the macroscopic density: $\rho^{-1 / 3}$. At low pressure, this length is roughly that of the potential-energy minimum, thus explaining why the latter has been generally assumed to be important.

The above considerations apply only for simple liquids; in general, both lengths play important roles for the physics. The irrelevance of any length defined by the microscopic potential emphasizes that the class of strongly correlating liquids is at the one end of the "complexity scale" where, at the other end, one finds systems like macromolecules, electrolytes, interfaces, micelles, or enzymes, for which multiple length scales are important [91].

\section{CONCLUDING REMARKS}

If you ask a chemist what is a simple liquid, he or she may likely answer that nonassociated liquids are simple, whereas associated liquids are generally much more complex. These two concepts are defined as follows in Chandler's textbook [13]. The intermolecular structure of a nonassociated liquid "can be understood in terms of packing. There are no highly specific interactions in these systems." In contrast, water is an example of an associated liquid, and its "linear hydrogen bonding tends to produce a local tetrahedral ordering that is distinct from what would be predicted by only considering the size and shape of the molecule" [13].

Packing usually refers to purely entropic, hard-spherelike behavior. Given that no realistic potentials are infinitely repulsive, it makes good sense to interpret packing more generally as all short-ranged effects of the intermolecular interactions. If one accepts this interpretation, the crucial role of the FCS for strongly correlating liquids is consistent with the understanding that the properties of nonassociated liquids can be interpreted in terms of packing: Once the forces from particles within the FCS are known, basically everything is known.

In other words, for a simple liquid there are no important long-range interactions, and "considering the size and shape of the molecule" [13] is enough to account for the liquid's physical properties. This applies even for the $r^{-4}$ IPL fluid, which one would a priori regard as systems with fairly long-ranged interactions.

The present definition of the class of simple liquids is thus consistent with the chemists' general picture of simple liquids. The new definition goes further, however, by quantifying simplicity via the virial potential-energy correlation coefficient $R$ of Eq. (2). In particular, simplicity is 
not an on/off property of the potential, but varies continuously with the state point. Thus, even a complex liquid like water is expected to approach simple behavior under sufficiently high pressure [76] and, conversely, the prototype strongly correlating LJ liquid becomes gradually more complex as density is lowered and the critical region and the gas phase are approached. Is this a problem, given that everyone agrees that the gas phase is simple? We do not think so. In fact, the gas phase is simple for an entirely different reason, namely, that molecules move freely most of the time, only interrupted by occasional fast and violent collisions with other molecules. It would be strange if a system exhibiting one form of simplicity could be transformed continuously in the phase diagram, maintaining its simplicity, into a system of an entirely different form of simplicity; one would expect the intermediate phase to be complex.

Liquid simplicity is characterized by the correlation coefficient $R$ of Eq. (2) being close to unity, i.e., that $1-R$ is a small number. This situation is typical in physics, where simplifying features always appear when some dimensionless number is small. The obvious question arises whether a statistical-mechanical perturbation theory may be constructed around simple liquids, embracing the more complex ones. Only time will tell whether this is possible, but it presents a challenge because the properties of IPL fluids $(R=1)$ cannot be worked out analytically.

A potentially annoying feature of defining liquid simplicity from the existence of strong correlations of the virial potential-energy fluctuations is that one cannot determine whether or not a given liquid is simple directly from the potential. We believe one should accept this as an acceptable cost for precisely defining the class of simple liquids. With the power of today's computers, this is much less of a problem than previously. For most systems, a brief simulation will determine whether or not the liquid is strongly correlating at the state point in question. Nevertheless, it would be nice to have an analytical criterion for liquid simplicity, i.e., for estimating whether $R>0.9$.

Except for IPL fluids, no system is simple in the entire fluid phase. This paper focused on the condensed liquid phase, not too far from the solid-liquid-coexistence line, but far from the critical point and the gas phase-it is here that some liquids are simple. The present focus on liquids is not meant to imply a limitation to the liquid phase, however. Simulations show that when a strongly correlating liquid crystallizes, the crystal is at least as strongly correlating [18]. A theory has been developed for (classical) strongly correlating crystals, showing that the property of strong virial potential-energy equilibrium fluctuations in the $N V T$ ensemble is an anharmonic effect that survives as $T \rightarrow 0$ [18]. Of course, low-temperature crystals are not classical systems, and for both liquids and crystals an interesting topic for future work is the implication of the proposed simplicity definition for the quantum description.

Section IVA summarized the several nominal essences of simple liquids. What is the real essence of liquid simplicity? Given that three fundamental characterizations of strongly correlating liquids are equivalent-the mathematical, the physical, and the new chemical (FCS) characterizations-this question cannot be answered unequivocally. At the end of the day, it is a matter of taste whether one defines liquid simplicity from the existence of strong virial potential-energy correlations, from the existence of isomorphs, from the existence of invariance curves in the phase diagram of constant-potential-energy hypersurfaces, or from the property that interactions beyond the FCS play little role.

\section{ACKNOWLEDGMENTS}

We gratefully acknowledge useful input from Livia Bove, Jesper Schmidt Hansen, Peter Harder, Mogens Niss, and Søren Toxværd. The centre for viscous liquid dynamics "Glass and Time" is sponsored by the Danish National Research Foundation (DNRF).

\section{APPENDIX A: MODEL DETAILS}

The model systems investigated are listed below. Quantities are given in rationalized units defined by putting $\epsilon=\sigma=1$. Masses that are not specified are unity.

Single-component inverse-power-law (IPL) fluids.$N=1024$ particles interacting via $v(r)=\epsilon(\sigma / r)^{n}$. Three different fluids were studied $(n=18,6,4)$.

Single-component Lennard-Jones liquid. $-N=1024$ particles interacting via Eq. (4).

Generalized Kob-Andersen binary mixture [28,29].-A binary mixture of $820 A$ particles and $204 B$ particles interacting via $v(r)=\epsilon_{\alpha \beta} /(12-n)\left[n\left(\sigma_{\alpha \beta} / r\right)^{12}-\right.$ $\left.12\left(\sigma_{\alpha \beta} / r\right)^{n}\right]$. Binary mixtures with $n=4,10$ were studied. The parameters used are $\epsilon_{A A}=1, \epsilon_{A B}=1.5$, $\epsilon_{B B}=0.5, \sigma_{A A}=2^{1 / 6}, \sigma_{A B}=0.8 \cdot 2^{1 / 6}, \sigma_{B B}=0.88 \cdot 2^{1 / 6}$.

Wahnstrom binary $L J$ mixture.-An equimolar binary mixture of $A$ and $B$ particles $(N=1024)$ interacting via the LJ potential. The parameters are $\epsilon_{A A}=\epsilon_{A B}=\epsilon_{B B}=$ $1, \sigma_{A A}=1, \sigma_{B B}=1 / 1.2, \sigma_{A B}=\left(\sigma_{A A}+\sigma_{B B}\right) / 2, m_{A}=$ $2, m_{B}=1$.

Buckingham liquid. $-N=1000$ particles interacting via $v(r)=\epsilon\left\{6 /(\alpha-6) \exp \left[\alpha\left(1-r / r_{m}\right)\right]-\alpha /(\alpha-6) \times\right.$ $\left.\left(r_{m} / r\right)^{6}\right\}$. The parameters used are $\epsilon=1, \alpha=14.5$, $r_{m}=2^{1 / 6}$.

Dzugutov liquid [51]. $-N=1024$ particles interacting via $v(r)=v_{1}+v_{2}$ where $v_{1}=\left\{A\left(r^{-n}-B\right) \exp [c /(r-a)]\right\}$ and $v_{2}=B \exp [d /(r-b)]$ and $r \geq a \Rightarrow v_{1}=0, r \geq$ $b \Rightarrow v_{2}=0(a<b)$. The parameters used are $a=1.87$, $b=1.94, \quad c=1.1, \quad d=0.27, \quad A=5.82, \quad B=1.28$, $n=16$. 
Lennard-Jones Gaussian liquid [52]. $-N=1024$ particles interacting via $v(r)=\epsilon\left\{(\sigma / r)^{12}-2(\sigma / r)^{6}-\right.$ $\left.\epsilon_{0} \exp \left[-\left(r-r_{0}\right)^{2} / 2 \sigma_{0}^{2}\right]\right\}$. The parameters used are $\sigma_{0}^{2}=$ $0.02, \epsilon_{0}=1.50, r_{0}=1.47$.

Gaussian core model [52]. $-N=1024$ particles interacting via $v(r)=\epsilon \exp \left[-(r / \sigma)^{2}\right]$.

The Hansen-McDonald molten salt model [57]. $-N=$ 2744 particles forming an equimolar binary mixture of singly charged cations and anions. The potential between two particles of charge $q_{\alpha}$ and $q_{\beta}$ is given by $v(r)=$ $(1 / 9) r^{-9}+q_{\alpha} q_{\beta} / r$, where $q_{+}=1, q_{-}=-1$.

Lewis-Wahnstrom OTP [58,59].-The LewisWahnstrom OTP model consists of three identical LJ particles rigidly bonded in an isosceles triangle with unity sides and a top angle of $75^{\circ}$ (number of molecules studied: $N=320$ ).

The asymmetric dumbbell model [19].-This molecular model consists of a large $(A)$ and a small $(B)$ LJ particle, rigidly bonded with a bond distance of $0.29 / 0.4963$ (number of molecules studied: 500). This model has $\sigma_{B B}=0.391 / 0.4963, \epsilon_{B B}=0.66944 / 5.726$, and $m_{B}=$ $15.035 / 77.106$. The $A B$ interaction between different molecules is determined by the Lorentz-Berthelot mixing rule.

Rigid SPC/E water [60].-This water model is an isosceles triangle with sides $1 / 3.166$ and base line 0.52 (number of molecules studied: 1000). The oxygen-oxygen intermolecular interactions are given by the LJ pair potential $\left(\epsilon_{\mathrm{OO}}=1, \sigma_{\mathrm{OO}}=1\right.$, and $\left.m_{\mathrm{O}}=16\right)$. There are no intermolecular LJ interactions for $\mathrm{H}-\mathrm{H}$ or $\mathrm{H}-\mathrm{O}$. The three particles are charged with $q_{\mathrm{O}}=-22.0$ and $q_{\mathrm{H}}=11.0$.

\section{APPENDIX B: HOW TO DELIMIT THE FIRST COORDINATION SHELL?}

In all simulations, the FCS cutoff was defined by placing the cutoff at the first minimum of the RDF, which is the standard definition of the FCS for liquids [13]. An alternative definition goes back to van der Waals [87]. The FCS is here identified with a sphere of radius determined by requiring that the average density $\rho_{\text {integrated }}$ within the FCS equals the overall average density $\rho_{\text {mean }}$. For the singlecomponent LJ liquid, this leads to virtually the same FCS [31]. Some change occurs for the standard KABLJ mixture, however. This is clear from Fig. 20 in which the integrated local density of $A$ particles calculated from the RDF (including the particle at the center) is shown as a function of the distance to the origin. The van der Waals distance is slightly larger than the first minimum of the RDF.

We applied this alternative definition of a FCS cutoff in Fig. 21, which shows the $A$-particle ISF for the $(12,6)$ KABLJ mixture of Fig. 20 simulated with, respectively, a cutoff at the first minimum of the RDF [Fig. 21(a)] and a van der Waals cutoff [Fig. 21(b)]. Although the difference is merely 0.05 , the van der Waals cutoff approx-

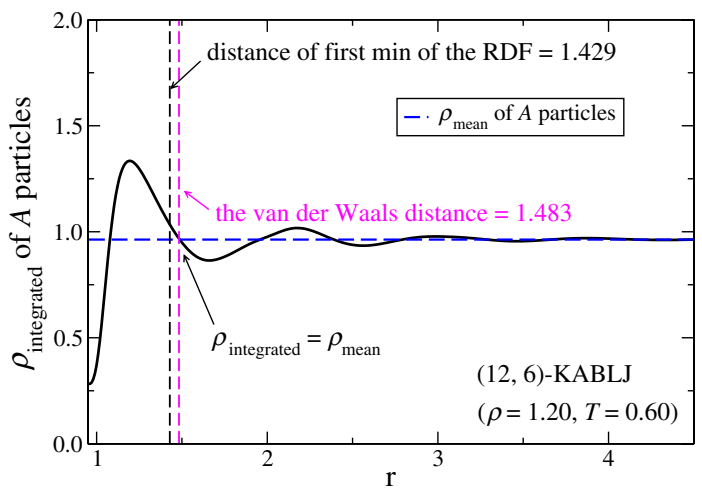

FIG. 20. Integrated local density $\rho_{\text {integrated }}$ of the $A$ particles of the standard KABLJ mixture plotted as a function of distance to the origin (calculated from the $A$-particle RDF). The dashed black line gives the distance of the minimum of the first peak of the RDF, the dashed magenta line gives the van der Waals distance, i.e., the distance at which the integrated local density equals the overall average density of the system. The horizontal dashed blue line marks the mean density $\rho_{\text {mean }}$ of $A$ particles in the simulated system $\left(\rho_{\text {mean }}=\right.$ $0.8 * 1.2)$.
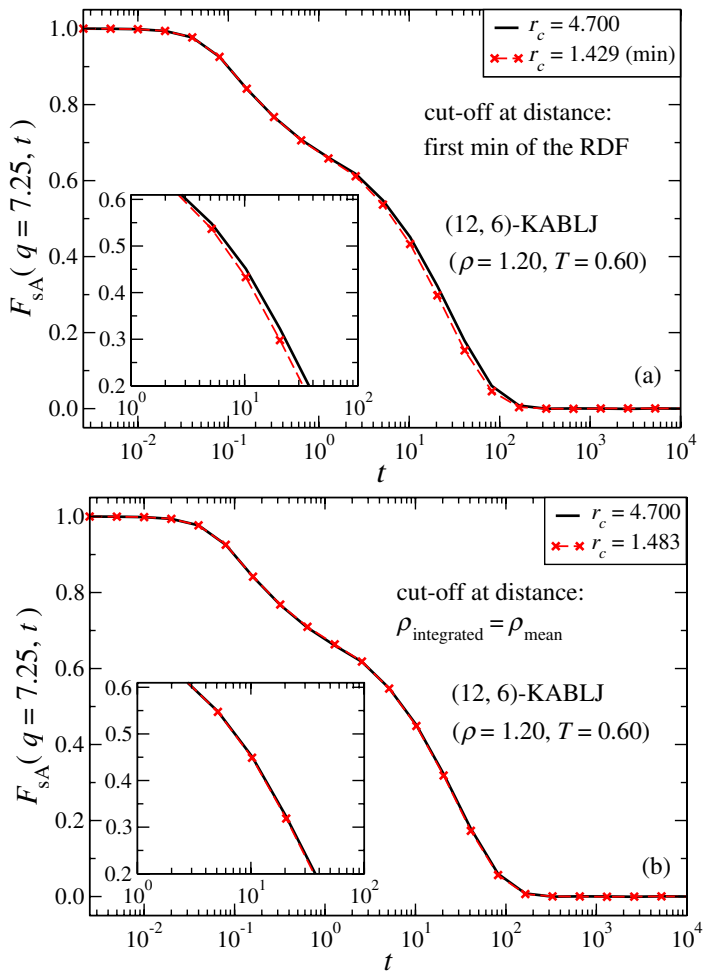

FIG. 21. Effect on the $A$-particle incoherent ISF for the standard KABLJ mixture of different of ways of delimiting the FCS. (a) FCS identified from the minimum beyond the first peak of the $A A$-particle RDF. (b) FCS identified by the van der Waals distance, i.e., the distance at which the integrated local density equals the mean density of the system. The van der Waals distance is slightly larger than the RDF minimum. Using this as defining the FCS-cutoff radius gives a better representation of the dynamics. 


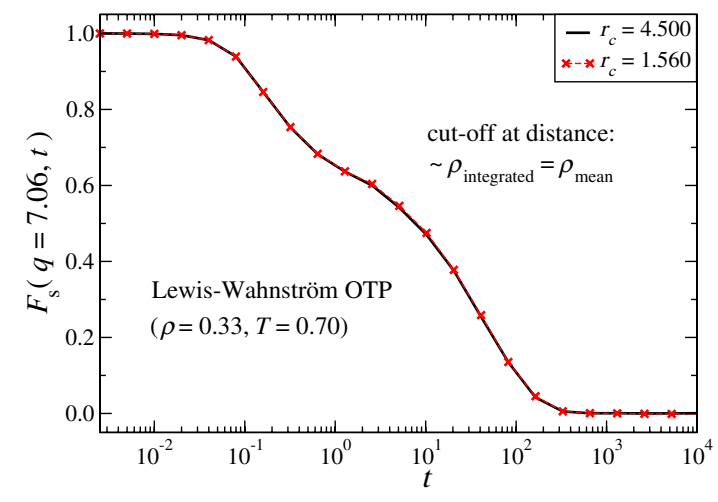

FIG. 22. Results for the incoherent ISF of the Wahnstrom OTP model with a cutoff at $r_{c}=1.56$ (red), and a large reference cutoff (black). The deviations of Fig. 17(b), in which $r_{c}=1.47$, disappear by choosing this slightly larger cutoff, not far from the van der Waals cutoff (1.53).

imates the reference ISF better than does the RDF minimum cutoff. Thus, it is possible that the van der Waals distance may serve as a better definition of the FCS than the standard FCS definition.

Identifying the exact size of the FCS for molecular systems is less straightforward, especially when different intermolecular interactions are involved. It is noteworthy how well the simple cutoff scheme in Fig. 18 represents the dynamics of the asymmetric dumbbell model. The slight deviations observed for the OTP model [Fig. 17(b)] disappear when the cutoff is increased from $r_{c}=1.47$ to $r_{c}=$ 1.56 (Fig. 22). This distance is close, but not identical, to the van der Waals distance calculated from the particle $\mathrm{RDF}$ (approximately 1.53). More work is needed to clarify the best way to delimit the FCS and define the FCS cutoff.

[1] Plato, Statesman (Dialogue), in Plato: Complete Works, edited by J. M. Cooper and D.S. Hutchinson (Hackett Publishing Co. Inc., Indianapolis, 1997).

[2] Aristotle, Categories (http://www.classicallibrary.org/ aristotle/categories/index.htm, 2001).

[3] J. Locke, An Essay Concerning Human Understanding, Book III (http://etext.lib.virginia.edu/modeng/modengL .browse.html, 2002).

[4] http://en.wikipedia.org/wiki/classification.

[5] J. L. Barrat and J. P. Hansen, Basic Concepts for Simple and Complex Liquids (Cambridge University Press, Cambridge, 2003).

[6] B. Kirchner, Theory of Complicated Liquids: Investigation of Liquids, Solvents and Solvent Effects with Modern Theoretical Methods, Phys. Rep. 440, 1 (2007).

[7] J. P. Hansen and J. R. McDonald, Theory of Simple Liquids (Academic, New York, 2005), 3rd ed.

[8] I.Z. Fisher, Statistical Theory of Liquids (University of Chicago, Chicago, 1964).

[9] S. A. Rice and P. Gray, The Statistical Mechanics of Simple Liquids (Interscience, New York, 1965).
[10] H. N. V. Temperley, J.S. Rowlinson, and G.S. Rushbrooke, Physics of Simple Liquids (Wiley, New York, 1968).

[11] N. K. Ailawadi, Equilibrium Theories of Classical Fluids, Phys. Rep. 57, 241 (1980).

[12] C. G. Gray and K. E. Gubbins, Theory of Molecular Fluids (Oxford University, New York 1984).

[13] D. Chandler, Introduction to Modern Statistical Mechanics (Oxford University, New York, 1987).

[14] U.R. Pedersen, N.P. Bailey, T.B. Schrøder, and J.C. Dyre, Strong Pressure-Energy Correlations in van der Waals Liquids, Phys. Rev. Lett. 100, 015701 (2008).

[15] U. R. Pedersen, T. Christensen, T. B. Schrøder, and J. C. Dyre, Feasibility of a Single-Parameter Description of Equilibrium Viscous Liquid Dynamics, Phys. Rev. E 77, 011201 (2008).

[16] N. P. Bailey, T. Christensen, B. Jakobsen, K. Niss, N. B. Olsen, U.R. Pedersen, T.B. Schrøder, and J.C. Dyre, Glass-Forming Liquids: One or More "Order" Parameters?, J. Phys. Condens. Matter 20, 244113 (2008).

[17] N. P. Bailey, U. R. Pedersen, N. Gnan, T. B. Schrøder, and J. C. Dyre, Pressure-Energy Correlations in Liquids. I. Results from Computer Simulations, J. Chem. Phys. 129, 184507 (2008).

[18] N. P. Bailey, U. R. Pedersen, N. Gnan, T. B. Schrøder, and J. C. Dyre, Pressure-Energy Correlations in Liquids. II. Analysis and Consequences, J. Chem. Phys. 129, 184508 (2008).

[19] T. B. Schrøder, U. R. Pedersen, N. P. Bailey, S. Toxvaerd, and J. C. Dyre, Hidden Scale Invariance in Molecular van der Waals Liquids: A Simulation Study, Phys. Rev. E 80, 041502 (2009).

[20] T. B. Schrøder, N. P. Bailey, U. R. Pedersen, N. Gnan, and J. C. Dyre, Pressure-Energy Correlations in Liquids. III. Statistical Mechanics and Thermodynamics of Liquids with Hidden Scale Invariance, J. Chem. Phys. 131, 234503 (2009).

[21] N. Gnan, T. B. Schrøder, U. R. Pedersen, N. P. Bailey, and J. C. Dyre, Pressure-Energy Correlations in Liquids. IV. "Isomorphs" in Liquid Phase Diagrams, J. Chem. Phys. 131, 234504 (2009).

[22] N. Gnan, C. Maggi, T. B. Schrøder, and J.C. Dyre, Predicting the Effective Temperature of a Glass, Phys. Rev. Lett. 104, 125902 (2010).

[23] U. R. Pedersen, T. B. Schrøder, and J. C. Dyre, Repulsive Reference Potential Reproducing the Dynamics of a Liquid with Attractions, Phys. Rev. Lett. 105, 157801 (2010).

[24] T. B. Schrøder, N. Gnan, U. R. Pedersen, N. P. Bailey, and J.C. Dyre, Pressure-Energy Correlations in Liquids. V. Isomorphs in Generalized Lennard-Jones Systems, J. Chem. Phys. 134, 164505 (2011).

[25] U. R. Pedersen, N. Gnan, N. P. Bailey, T. B. Schrøder, and J.C. Dyre, Strongly Correlating Liquids and their Isomorphs, J. Non-Cryst. Solids 357, 320 (2011).

[26] L. Berthier and G. Tarjus, Nonperturbative Effect of Attractive Forces in Viscous Liquids, Phys. Rev. Lett. 103, 170601 (2009).

[27] L. Berthier and G. Tarjus, The Role of Attractive Forces in Viscous Liquids, J. Chem. Phys. 134, 214503 (2011). 
[28] W. Kob and H.C. Andersen, Testing Mode-Coupling Theory for a Supercooled Binary Lennard-Jones Mixture I: The van Hove Correlation Function, Phys. Rev. E 51, 4626 (1995).

[29] W. Kob and H.C. Andersen, Testing Mode-Coupling Theory for a Supercooled Binary Lennard-Jones Mixture. II. Intermediate Scattering Function and Dynamic Susceptibility, Phys. Rev. E 52, 4134 (1995).

[30] D. Weeks, D. Chandler, and H.C. Andersen, Role of Repulsive Forces in Determining the Equilibrium Structure of Simple Liquids, J. Chem. Phys. 54, 5237 (1971).

[31] S. Toxvaerd and J.C. Dyre, Communication: Shifted Forces in Molecular Dynamics, J. Chem. Phys. 134, 081102 (2011).

[32] S. Toxvaerd and J. C. Dyre, Role of the First Coordination Shell in Determining the Equilibrium Structure and Dynamics of Simple Liquids, J. Chem. Phys. 135, 134501 (2011).

[33] A. Paliwal, D. Asthagir, L. R. Pratt, H. S. Ashbaugh, and M.E. Paulaitis, An Analysis of Molecular Packing and Chemical Association in Liquid Water Using Quasichemical Theory, J. Chem. Phys. 124, 224502 (2006).

[34] M.P. Allen and D. J. Tildesley, Computer Simulation of Liquids (Oxford Science Publications, New York, 1987).

[35] T. Ree, H.S. Kang, C.S. Lee, and F.H. Ree, A Perturbation Theory of Classical Equilibrium Fluids, J. Chem. Phys. 82, 414 (1985).

[36] R. W. Hall and P. G. Wolynes, Intermolecular Forces and the Glass Transition, J. Phys. Chem. B 112, 301 (2008).

[37] C. J. Fennell and J. D. Gezelter, Is the Ewald Summation Still Necessary? Pairwise Alternatives to the Accepted Standard for Long-Range Electrostatics, J. Chem. Phys. 124, 234104 (2006).

[38] J. S. Hansen, T. B. Schrøder, and J. C. Dyre, Simplistic Coulomb Forces in Molecular Dynamics: Comparing the Wolf and Shifted-Force Approximations, arXiv:1108.5267.

[39] S. Nosé, A Unified Formulation of the Constant Temperature Molecular Dynamics Methods, J. Chem. Phys. 81, 511 (1984).

[40] W. G. Hoover, Canonical Dynamics: Equilibrium Phase-Space Distributions, Phys. Rev. A 31, 1695 (1985).

[41] D. Frenkel and B. Smit, Understanding Molecular Simulation (Academic, New York, 2002).

[42] All simulations were performed using a molecular dynamics code for NVIDIA graphics cards, which is available as open source at http://rumd.org.

[43] S. Toxvaerd, Algorithms for Canonical Molecular Dynamics Simulations, Mol. Phys. 72, 159 (1991).

[44] T. Ingebrigtsen, O.J. Heilmann, S. Toxvaerd, and J.C. Dyre, Time Reversible Molecular Dynamics Algorithms with Holonomic Bond Constraints in the NPH and NPT Ensembles Using Molecular Scaling, J. Chem. Phys. 132, 154106 (2010).

[45] S. Toxvaerd, O. J. Heilmann, T. Ingebrigtsen, T. B. Schrøder, and J.C. Dyre, Time-Reversible Molecular Dynamics Algorithms with Bond Constraints, J. Chem. Phys. 131, 064102 (2009).
[46] M. E. Fisher and D. Ruelle, The Stability of Many-Particle Systems, J. Math. Phys. (N.Y.) 7, 260 (1966).

[47] M. Baus and J. P. Hansen, Statistical Mechanics of Simple Coulomb Systems, Phys. Rep. 59, 1 (1980).

[48] G. Wahnström, Molecular-Dynamics Study of a Supercooled Two-Somponent Lennard-Jones System, Phys. Rev. A 44, 3752 (1991).

[49] R. A. Buckingham, The Classical Equation of State of Gaseous Helium, Neon, and Argon, Proc. R. Soc. A 168, 264 (1938).

[50] A. A. Veldhorst, L. Bøhling, J.C. Dyre, and T. B. Schrøder, Isomorphs in the Phase Diagram of a Model Liquid without Inverse Power Law Repulsion, Eur. Phys. J. B 85, 24 (2012).

[51] M. Dzugutov, Glass Formation in a Simple Monatomic Liquid with Icosahedral Inherent Local Order, Phys. Rev. A 46, R2984 (1992).

[52] M. Engel and H.R. Trebin, Self-Assembly of Monatomic Complex Crystals and Quasicrystals with a Double-Well Interaction Potential, Phys. Rev. Lett. 98, 225505 (2007).

[53] V. V. Hoang and T. Odagaki, Glasses of Simple Liquids with Double-Well Interaction Potential, Physica B (Amsterdam) 403, 3910 (2008).

[54] F.H. Stillinger, Phase Transitions in the Gaussian Core System, J. Chem. Phys. 65, 3968 (1976).

[55] F. H. Stillinger and T. A. Weber, Amorphous State Studies with the Gaussian Core Model, J. Chem. Phys. 70, 4879 (1979).

[56] A. Ikeda and K. Miyazaki, Glass Transition of the Monodisperse Gaussian Core Model, Phys. Rev. Lett. 106, 015701 (2011).

[57] J. P. Hansen and I. R. McDonald, Statistical Mechanics of Dense Ionized Matter. IV. Density and Charge Fluctuations in a Simple Molten Salt, Phys. Rev. A 11, 2111 (1975).

[58] G. Wahnström and L.J. Lewis, Molecular Dynamics Simulation of a Molecular Glass at Intermediate Times, Physica A (Amsterdam) 201, 150 (1993).

[59] L. J. Lewis and G. Wahnström, Molecular-Dynamics Study of Supercooled Ortho-Terphenyl, Phys. Rev. E 50, 3865 (1994).

[60] H. J. C. Berendsen, J. R. Grigera, and T. P. Straatsma, The Missing Term in Effective Pair Potentials, J. Phys. Chem. 91, 6269 (1987).

[61] C. M. Roland, S. Hensel-Bielowka, M. Paluch, and R. Casalini, Supercooled Dynamics of Glass-Forming Liquids and Polymers under Hydrostatic Pressure, Rep. Prog. Phys. 68, 1405 (2005).

[62] T.S. Ingebrigtsen, T. B. Schrøder, and J.C. Dyre, Isomorphs in Model Molecular Liquids, J. Phys. Chem. B 116, 1018 (2012).

[63] S. Torquato, T. M. Truskett, and P. G. Debenedetti, Is Random Close Packing of Spheres Well Defined? Phys. Rev. Lett. 84, 2064 (2000).

[64] T. M. Truskett, S. Torquato, and P. G. Debenedetti, Towards a Quantification of Disorder in Materials: Distinguishing Equilibrium and Glassy Sphere Packings, Phys. Rev. E 62, 993 (2000).

[65] J.R. Errington and P. G. Debenedetti, Relationship between Structural Order and the Anomalies of Liquid Water, Nature (London) 409, 318 (2001). 
[66] M. S. Shell, P. G. Debenedetti, and A. Z. Panagiotopoulos, Molecular Structural Order and Anomalies in Liquid Silica, Phys. Rev. E 66, 011202 (2002).

[67] J. R. Errington, P. G. Debenedetti, and S. Torquato, Quantification of Order in the Lennard-Jones System, J. Chem. Phys. 118, 2256 (2003).

[68] K. L. Ngai, R. Casalini, S. Capaccioli, M. Paluch, and C.M. Roland, Do Theories of the Glass Transition, in which the Structural Relaxation Time Does not Define the Dispersion of the Structural Relaxation, Need Revision? J. Phys. Chem. B 109, 17356 (2005).

[69] T. S. Ingebrigtsen, L. Bøhling, T. B. Schrøder, and J. C. Dyre, Communication: Thermodynamics of Condensed Matter with Strong Pressure-Energy Correlations, J. Chem. Phys. 136, 061102 (2012).

[70] S. A. Khrapak and G. E. Morfill, Accurate Freezing and Melting Equations for the Lennard-Jones System, J. Chem. Phys. 134, 094108 (2011).

[71] L. Bøhling, T.S. Ingebrigtsen, A. Grzybowski, M. Paluch, J.C. Dyre, and T.B. Schrøder, Beyond Power-Law Density Scaling: Theory, Simulation, and Experiment, arXiv:1112.1602.

[72] D. Coslovich and C.M. Roland, Density Scaling in Viscous Liquids: From Relaxation Times to Four-Point Susceptibilities, J. Chem. Phys. 131, 151103 (2009).

[73] L. Pauling, The Nature of the Chemical Bond (Cornell University, Ithaca, 1939).

[74] L. E. Bove, F. Sacchetti, C. Petrillo, B. Dorner, F. Formisano, and F. Barocchi, Neutron Investigation of the Ion Dynamics in Liquid Mercury: Evidence for Collective Excitations, Phys. Rev. Lett. 87, 215504 (2001).

[75] C. Petrillo, F. Sacchetti, E. Guarini, L. E. Bove, and F. Demmel, Collective Modes in a Saturated LithiumAmmonia Solution as a Probe of the Response of the Low-Density Homogeneous Electron Gas, Phys. Rev. B 84, 094206 (2011).

[76] J. J. Papini, T. B. Schrøder, and J. C. Dyre, Do All Liquids Become Strongly Correlating at High Pressure? arXiv:1103.4954.

[77] J. Mittal, J.R. Errington, and T.M. Truskett, Thermodynamics Predicts How Confinement Modifies the Dynamics of the Equilibrium Hard-Sphere Fluid, Phys. Rev. Lett. 96, 177804 (2006).

[78] J. Mittal, J. R. Errington, and T. M. Truskett, Relationships Between Self-Diffusivity, Packing Fraction, and Excess
Entropy in Simple Bulk and Confined Fluids, J. Phys. Chem. B 111, 10054 (2007).

[79] G. Goel, W.P. Krekelberg, J. R. Errington, and T. M. Truskett, Tuning Density Profiles and Mobility of Inhomogeneous Fluids, Phys. Rev. Lett. 100, 106001 (2008).

[80] R. Chopra, T. M. Truskett, and J.R. Errington, ExcessEntropy Scaling of Dynamics for a Confined Fluid of Dumbbell-Shaped Particles, Phys. Rev. E 82, 041201 (2010).

[81] J. D. Weeks, K. Vollmayr, and K. Katsov, Intermolecular Forces and the Structure of Uniform and Nonuniform Fluids, Physica A (Amsterdam) 244, 461 (1997).

[82] J.D. Weeks, Connecting Local Structure to Interface Formation: A Molecular Scale van der Waals Theory of Nonuniform Liquids, Annu. Rev. Phys. Chem. 53, 533 (2002).

[83] Z. Yan, S. V. Buldyrev, P. Kumar, N. Giovambattista, P. G. Debenedetti, and H. E. Stanley, Structure of the First- and Second-Neighbor Shells of Simulated Water: Quantitative Relation to Translational and Orientational Order, Phys. Rev. E 76, 051201 (2007).

[84] W.P. Krekelberg, J. Mittal, V. Ganesan, and T. M. Truskett, How Short-Range Attractions Impact the Structural Order, Self-Diffusivity, and Viscosity of a Fluid, J. Chem. Phys. 127, 044502 (2007).

[85] W.P. Krekelberg, J. Mittal, V. Ganesan, and T. M. Truskett, Structural Anomalies of Fluids: Origins in Second and Higher Coordination Shells, Phys. Rev. E 77, 041201 (2008).

[86] W. Hujo, B. S. Jabes, V. K. Rana, C. Chakravarty, and V. Molinero, The Rise and Fall of Anomalies in Tetrahedral Liquids, J. Stat. Phys. 145, 293 (2011).

[87] J.S. Rowlinson and B. Widom, Molecular Theory of Capillarity (Clarendon, Oxford, 1982).

[88] B. Widom, Intermolecular Forces and the Nature of the Liquid State, Science 157, 375 (1967).

[89] J.A. Barker and D. Henderson, What is "Liquid"? Understanding the States of Matter, Rev. Mod. Phys. 48, 587 (1976).

[90] S. Zhou and J. R. Solana, Progress in the Perturbation Approach in Fluid and Fluid-Related Theories, Chem. Rev. 109, 2829 (2009).

[91] B. Bagchi and C. Chakravarty, Interplay Between Multiple Length and Time Scales in Complex Chemical Systems, J. Chem. Sci. 122, 459 (2011). 